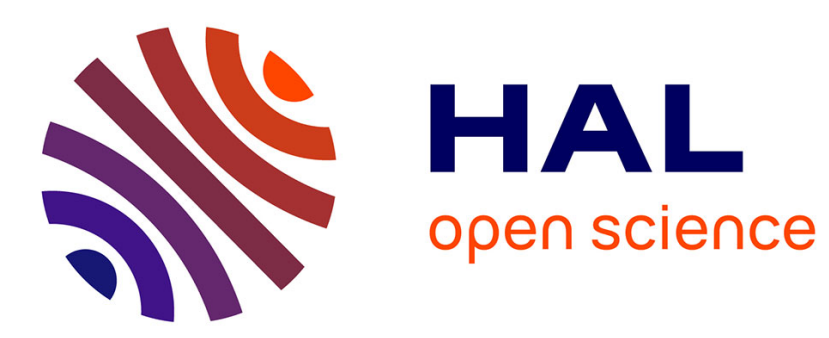

\title{
MAX JACOB ET LES ARTS DE LA SCENE: ORIENTATIONS BIBLIOGRAPHIQUES
}

Patricia Sustrac

\section{To cite this version:}

Patricia Sustrac. MAX JACOB ET LES ARTS DE LA SCENE: ORIENTATIONS BIBLI-

OGRAPHIQUES. Les Cahiers Max Jacob, 2019, LES CAHIERS MAX JACOB, 19/20. hal-02314733

\section{HAL Id: hal-02314733 \\ https://hal.science/hal-02314733}

Submitted on 14 Oct 2019

HAL is a multi-disciplinary open access archive for the deposit and dissemination of scientific research documents, whether they are published or not. The documents may come from teaching and research institutions in France or abroad, or from public or private research centers.
L'archive ouverte pluridisciplinaire HAL, est destinée au dépôt et à la diffusion de documents scientifiques de niveau recherche, publiés ou non, émanant des établissements d'enseignement et de recherche français ou étrangers, des laboratoires publics ou privés. 


\title{
MAX JACOB ET LES ARTS DE LA SCÈNE : ORIENTATIONS BIBLIOGRAPHIQUES
}

Patricia SUSTRAC*

\begin{abstract}
$\mathrm{N}$ ous mentionnons dans cette section les recueils de Max Jacob consacrés au arts de la scène ainsi que des études critiques. Les articles sont classés par ordre alphabétique d'auteur ; les ouvrages, entièrement ou partiellement consacrés aux genres, les travaux universitaires ou numéros spéciaux sont classés à part. Nous indiquons également les ressources archivistiques des collections publiques.

Concernant les transpositions musicales des poèmes de l'auteur, nous avons choisi de recenser uniquement les éditions princeps et les premières auditions. Concernant les adaptations posthumes, nous renvoyons au site de la Sacem ${ }^{1}$. Cette orientation bibliographique est donc volontairement non exhaustive, notre travail a porté essentiellement sur une clarification des sources. Pour le théâtre, outre le recensement bibliographique, nous indiquons les créations des théâtres et compagnies subventionnés uniquement.

Concernant la correspondance avec des artistes nous renvoyons à la bibliographie de l'épistolaire que nous avions établie (CMJ,13/14), nous mentionnons ici les correspondances particulières parues en volume ou encore inédites
\end{abstract}

\footnotetext{
* $\quad$ Patricia Sustrac a publié des articles critiques et biographiques, des bibliographies et édité plusieurs correspondances de Max Jacob. Elle est Présidente de l'Association des Amis de Max Jacob depuis 2005, Directrice de publication et Secrétaire de rédaction des Cahiers Max Jacob.
} 
conservées en fonds publics ; nous avons écarté les parutions en revues à l'exception des cahiers ou revues consacrés à Jacob. Pour chacune des publications, lorsque le lieu d'édition est Paris, celui-ci n'est pas indiqué.

\section{THÉÂTRE : PUBLICATIONS ANTHUMES}

« «Les Maladies de foie», Les Feux de Paris, n 3, février-mars 1936. - rééd. Max Jacob and Les Feux de Paris, unpublished letters from Max Jacob to Jean Fraysse, correspondance présentée par Neal Oxenhandler, Berkeley/Los Angeles : University of California Press, coll. University of California Publications in Modern philology, vol. 35, n 4, 1964, p. 221-283. - rééd. L'Échelle de Jacob, recueil d'inédits réunis et présentés par Nicole et José-Emmanuel Cruz, Lausanne : La Bibliothèque des Arts, coll. Pergamine, 1994, p. 97-102.

« « Le Veuf (fragment)», Le Mail, n 5, 1928, p. 252-257.

Le Roi de Béotie, Gallimard, in-18, 250 p., 10 déc. 1921. Tirage : 120 ex. in-4 Tellière sur papier Vergé Lafuma-Navarre au filigrane de $L a N R F$ dont 8 ex. $\mathrm{HC}$ marqués de $\mathrm{A}$ à $\mathrm{H}, 100$ ex. réservés aux bibliophiles de $L a N R F, \mathrm{n}^{\circ} \mathrm{I}$ à $\mathrm{C}$, 12 ex. $n^{\circ} \mathrm{CI}$ à CXII et 840 ex. sur papier Vélin pur fil Lafuma Navarre dont 10 ex. HC marqués a à j, 800 ex. réservés aux amis de l'édition originale $\mathrm{n}^{\circ} 1$ à 800, 30 ex. d'auteur $\mathrm{HC} \mathrm{n}^{\circ} 801$ à 830.- rééd. [La Couronne de Vulcain et Histoire du roi Kaboul $1^{e r}$ et du marmiton Gauwain] : Gallimard, 1971.- rééd. O., 835-952.

Dédicace de l'œuvre : «À toi qui que tu sois qui achète mes livres songe que d'un auteur c'est la clef qu'on te livre. »

Contient trois pièces : "Chantage »; « Entrepôt Voltaire, gros et détail, articles en tous genres »; " Entrepôt Voltaire : la facture en anglais ».

\& Dos d'Arlequin, éd. Sagittaire-Simon Kra², 30 avril 1921, in-8, 77 p., 20 illustrations de l'auteur dont 3 en couleurs, l'ensemble gravé sur bois (11 dessins, 9 vignettes), imp. Ducros, Lefèvre et Colas. Tirage : 10 ex. sur Japon avec une suite de bois sur papier de Chine, ex. illustrés marginalement de dessins de Max Jacob, $\mathrm{n}^{\circ} 1$ à $10 ; 25$ ex. sur Japon avec une suite de bois sur papier de Chine, $\mathrm{n}^{\circ} 11$ à $35 ; 25$ ex. HC sur Vélin pur fil et 12 ex. sur papier de Chine $\mathrm{n}^{\circ} 36$ à 47 et souscrits par Messieurs Ronald Davis et \& $\mathrm{C}^{\mathrm{ie}}, 173$, rue de Courcelles, Paris. 
Contient trois pièces : «L'Auteur au théâtre, petit drame portatif »; « Don Juan $^{3} »$; « Le Divorce de la duchesse de Prazzel ».

« « Histoire de Don Juan », Nord-Sud, 15 mars 1917, p. 13-15.

\section{THÉÂTRE, THÉÂTRE-BOUFFE : PUBLICATIONS POSTHUMES}

\& Les Pétroles du Béloutchistan, voir la publication dans le présent volume.

\& Ruffian toujours, truand jamais, voir la publication dans le présent volume.

\& « Paris-Province, dialogues, Petit dialogue : peintre et marchand », " Le Journal des Modes ou les ressources de Florimond », Max Jacob et la création. Actes du colloque d'Orléans, 4-5 mars 1994, ville et l'Université d'Orléans : éd. J.-Michel Place, coll. Surfaces, 2000, p. 97-132.

\& Le Terrain Bouchaballe, comédie en 3 actes présentée par Roger Secrétain, notes d'Hélène Henry, Mortemart : éd. Rougerie, 1982.

\& La Sainte Hermandade, pièce en 1 acte, Mithra, n 1, 1951, p. 1-7. - rééd. La $N R F$, nov. $1955, \mathrm{n}^{\circ} 35$, p. 1009-1017.

\& Un amour du Titien, opérette inspirée en 5 actes et 4 tableaux, [partition d'Henri Sauguet inachevée], suivi de La Police napolitaine, pièce en 1 acte, $C M J / 3,1953$, p. 9-72 et 73-96 [précédé de [Anonyme], « Le Théâtre de Max Jacob »; SAUGUET Henri, "Quand j'écrivais une opérette avec Max Jacob», p. 7-8].

\& Les Contrebandiers, opéra-bouffe, Biblio, bibliographie-littérature, XVII année, $n^{\circ}$ 1, janv. 1949, p. 3-5 [p. 5, facsimile d’un f. légendé : «Opéra-bouffe écrit par Max Jacob pour Georges Auric », ms non retrouvé].

\& Aristide, pièce en 1 acte, Le Milieu du siècle, éd. B. Janin, 1947, n 1, p. 203209 [version dramatique de la lettre fictive «Ils en ont ri ensemble au café », O., 994]. 


\section{OEUVRES DE COLLABORATION ${ }^{4}$}

\& JACOB Max, LAGARDE Pierre, «Projet d'auteurs », Comœdia, 8 avril 1934, La Turquoise (ms perdu).

\& JACOB Max, OLIN Marcel, sketch, (6 oct. 1922, à Georges Gabory, AAI, 112).

\& ALLIER Pierre, JACOB Max, Kemper-Cancans, 1910, voir infra.

\section{MAX JACOB LIBRETTISTE}

\& JACOB Max, SAUGUET Henri, Les Pénitents en maillots roses, piano et voix, mars 1944, éd. Heugel, 1949. Poèmes issus du recueil éponyme : «Ports de l'enfer » $(O ., 685)$; « À une sainte le jour de sa fête » $(O ., 695)$; « Jardin mystérieux » $(O ., 698) ;$ « Marine à Roscoff » $(O ., 697) ;$ « La Ville» $(O ., 705)$.

« ACKERMANS Hippolyte, JACOB Max, «Chanson » (O., 1619), dossier Sacem du 31 janvier $1938^{5}$, la partition non éditée .

\& JACOB Max, NABOKOV Nicolas, L'Aubépin et cinq autres mélodies, piano et voix, éd. la Sirène musicale, 1938 (textes français, allemand et anglais ${ }^{6}$ ) : L'Aubépin (Le Cornet à dés II - une suite : La Différence, coll. Orphée, 1994, p. 44) ; « Angoisses et autres » (O., 1413-1414), « L'Adultère ${ }^{7}$ », « Lune ${ }^{8} . »$

\& CASABIANCA René, JACOB Max, "L’homme est menteur, chanson populaire ». Dossier Sacem août 1933, partition non éditée. Voir infra dossier CMJ 19/20.

\& JACOB Max, RIETI Vittorio, Quatre poèmes de Max Jacob, piano et voix, 1933 : « Le Noyer fatal» (Actualités éternelles, La Différence, 1994, p. 223); Poèmes inédits : « Vos yeux $\operatorname{clos}^{9} »$, « La crise ${ }^{10} »$, « Monsieur le duc » (Voir infra dossier CMJ 19/20).

\& JACOB Max, POULENC Francis, Cinq poèmes de Max Jacob, piano et voix, 1932. Poèmes rassemblés posthumément dans Morven le Gaélique : «Chanson» $(O ., 1620)$, « Cimetière » $(O ., 1621)$, « La Petite Servante » $(O$. , 1628), « Berceuse de la petite servante » $(O ., 1637)$, « Chanson » $(O ., 1668)$. 
\& JACOB Max, POULENC Francis, Le Bal masqué ${ }^{11}$, cantate profane pour baryton (ou mezzo) et orchestre de chambre, Rouart, Lerolle et $\mathrm{C}^{\mathrm{ie}}$, éd. Salabert, 1948. Euvre dédiée aux commanditaires M. et Mme le Vte et la Vtesse de Noailles, 20 avril 1932, Théâtre de Hyères. Orchestre : Roger Désormières ; piano : Francis Poulenc; décors : Christian Bérard (Laboratoire central : $O ., 620-625)$.

\& JACOB Max, NABOKOV Nicolas, Collectionneurs d'échos ${ }^{12}$. Petite cantate pour soprano et basse, le chœur du public, neuf instruments et batterie ; œuvre dédiée aux commanditaires M. et Mme le Vte et la Vtesse de Noailles, 20 avril 1932, Théâtre de Hyères. Soprano : Madeleine Vitha ; Basse : GilbertMoryn.

\& JACOB Max, THOMSON Virgil, Stabat Mater, cantate pour soprano et quatuor à cordes, juin $1931^{13}$, Londres : Arrow Press, 1933. Poème éponyme publié posthumément dans Morven le Gaélique (O., 1637).

\& JACOB Max, POULENC Francis, Cinq poèmes, voix et piano, éd. Salabert, 1931, Conservatoire de Paris. Poèmes (dédiés par le musicien lors de la création) rassemblées posthumément dans Morven le Gaélique : "Chanson bretonne ", $(O ., 1620$, à M.- Blanche de Polignac) ; "Cimetière » $(O ., 621$, à Madeleine Vitha); "La Petite servante» $(O ., 1628$, à Suzanne Peignot); « Berceuse de la petite servante» $(O ., 1637$, à Suzanne Balguerie) ; « Souric et Mouric » (O., 1668 à Ève Curie).

\& JACOB Max, BORDES Francis, "Fable sans moralité », piano et voix, éd. Maurice Sénart, 1931 : Cornet à dés (O., 394).

« JACOB Max, JOLIVET André, « La mule de Lord Bolingbroke », piano et voix, 1930 («Ennui sur le taureau d'Europe », O., 679-680).

\& JACOB Max, POULENC Francis, Quatre poèmes de Max Jacob, œuvre pour ténor, flûte, hautbois, basson et clarinette, 1921, création 7 janvier 1922, lieu non précisé. Poèmes repris dans Les Pénitents en maillots roses (1925) : " Ennui sur le taureau d'Europe », (op. cit.), «Intérimes », (O., 675-676), « Passé et présent », (O., 568-569), « Poèmes burlesques [Dans le buisson de mimosas] », (O., 689). 
\& JACOB Max, ROLAND-MANUEL ${ }^{14}$, "Air de Pantalon, $1^{\text {er }}$ acte », La Revue musicale, texte et partition extrait de l'opéra-bouffe éponyme : $L a$ $N R F, 2^{\mathrm{e}}$ année, $\mathrm{n}^{\circ} 6$, T. III, suppl. musical ${ }^{\mathrm{er}}$ avril 1921.- rééd. : Au Ménestrel, Heugel, 1922, partition pour chant et piano, 88 p. Création au Trianon Lyrique (6-15 déc. $\left.1922^{15}\right)$. Orchestre dirigé par d'André Caplet ; dir. de scène : Léon Joubert; chef de chant : Hélène Léon ; chef de chœurs : MM. Clément et Bonnafé ; décors : Maxime Dethomas réalisés par MM. Mouveau, Deshays et Arnaud. Distribution : Isabelle, M ${ }^{\text {elle }}$ Évrard ; Zerbinette, Andrée Moreau ; Pantalon: Mario ; Arlequin: Alex Jouvin ; Pierrot : de Trévi.

\& HONEGGER Arthur, JACOB Max, La Mort de Sainte Alméenne, mystère musical en 1 acte et 2 tableaux. Orchestration inachevée initiée circa 1918. Intermède interprété le 25 nov. 1920, lieu non précisé, compte rendu de Darius Milhaud ${ }^{16}$, décors de Guy-Pierre Fauconnier ${ }^{17}$. Quatre personnages : Sainte Alméenne (soprano), l'Ange (soprano), la Sœur (mezzo-soprano), le jeune homme (baryton). $1^{\text {er }}$ tableau : un jardin dans un cloître (4 scènes), $2^{\mathrm{e}}$ tableau : la cellule $(2 \text { scènes })^{18}$.

\section{TEXTES D'ESTHÉTIQUE}

« «À Propos de théâtre », voir la publication dans le présent volume.

« « Note à propos des Beaux-Arts », Philosophies, 15 mars 1924, n 1, p. 1-5.

\& «Théâtre et Cinéma », Nord-Sud, fév. 1918, n 12, p. 9-12 - rééd. O., 1392-1395.

\section{TEXTES DE FICTION, TEXTES POÉTIQUES, ARTICLES, TÉMOIGNAGES, HOMMAGES}

« « Louis Armstrong (Départ du château)», Jazz hot, n 13, déc. 1936, p. 11. rééd. voir infra dossier CMJ 19/20.

« « Mes impressions de scène », Les Feux de Paris, n 3, février-mars 1936. rééd. Max Jacob and Les Feux de Paris, op. cit., p. 281-283.

« «Lettre d'une jeune aspirante "star" et sa réponse », Les Cahiers du film, revue française du cinéma français ${ }^{19}, \mathrm{n}^{\circ}$ 1, 15 déc. 1934, p. 12-13. - rééd. CMJ/2, mars 1952, p. 31-33; Cinéma, n 51, nov. 1960, p. 52-53. 
« « Cinéma (collection d'autographes de Max Jacob) », Heim ${ }^{20}, \mathrm{n}^{\circ}$ 10, oct. 1934, p. 16-17 (sous alias de Jean Capain). - rééd. CMJ/2, op. cit., p. 25-27; Le Cabinet noir sous le titre « Lettre du figurant qui fait du cinéma » : Gallimard, $1968 ;$ O., p. 1079-1080.

\& «Le Cinéma et le révolver », Mouvement, $\mathrm{n}^{\circ}$ 1, juin 1933, p. 25.

« « Élucubration », Les Chroniques du jour, n 7-8, déc. 1926, p. 15-16.- rééd. Maintenant, Cahiers d'art et de littérature, ${ }^{\circ} 3$, juillet 1946, p. 240-242 [ms reproduit avec mention du dédicataire : Maurice Sachs].

ه "L'Humour est la danse sur le volcan », Le Disque vert, p. 35-37. - rééd. 1924, Louis Émié, Dialogues avec Max Jacob : Corrêa/Buchet-Chastel, coll. Mises au point, 1954, p. 231-233. - rééd. Quatre problèmes à résoudre, exercices proposés aux candidates au grade du Brevet élémentaire, Liège : Pierre Aelberts éd. Dynamo, 1962, p. 10-12.- rééd. Louis Émié, Dialogues avec Max Jacob, Bordeaux : Le Festin, 1994, p. 214.

« « L'acteur de cinéma ivre ${ }^{21} », 1923$, voir infra.

« « Petite chronique cinématographique », L'Élan, n 10, déc. 1916, p. 1.

\& «Saoul-titre : Charlot s'amuse ou Charlot et Racine », 291, New-York, n 12, fév. 1916, p. 4. - rééd. CES 8, 1986, p. 32-36.

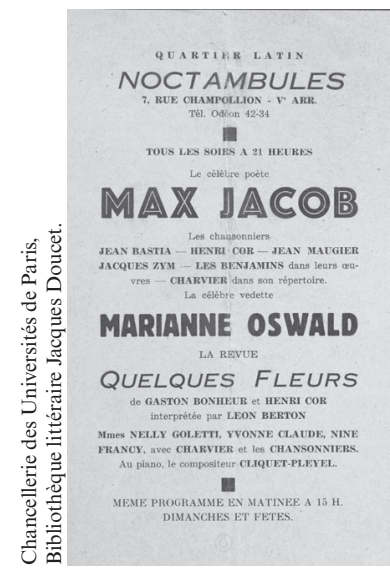

\section{REPRÉSENTATIONS SCÉNIQUES}

\& 21 mars-13 avril 1936, Les Noctambules, 7 rue Champollion, Paris ${ }^{22}$.

\& Presse : voir infra Médiathèque des Ursulines, Quimper

œ Euvres graphiques : voir infra Musée des Beaux-arts d'Orléans et «Impressions de scène » : voir supra.

\& 3 avril 1933, 9 h 30 à 11 h., au Bœuf sur le toit, « Soirée Max Jacob ${ }^{23}$. » Organisateur : Compagnie Le Rideau de 
Paris (Marcel Herrand et Jean Marchand dir.). Programme : causerie de Pierre Guéguen ; mélodies sur des poèmes de Max Jacob par Jane Bathori ${ }^{24}$. Musique de Roland-Manuel, Nabokov, Poulenc, Rieti et Sauguet (des extraits d'Un Amour du Titien selon Raphaël Cluzel, voir infra). Poèmes de Max Jacob, exposition et signature.

\& 31 mai et/ou 2 juin 1930, Théâtre de l'Atelier, place Dancourt (dir. Charles Dullin), Le Kleptomane, sketch en 1 acte et 1 tableau, lecture théâtralisée ou mise en scène par Julien Lanoë ${ }^{25}$.

\& 1927-1940, Matinées Poétiques ${ }^{26}$, Comédie-Française, comédiens pas toujours précisés ; 30 avril 1927, 14 janvier 1928 : «Poème »- sans autres précisions -, « Jeux sur le mot "caste" » $(O ., 384)$; 18 janvier 1930 : «Honneur de la Sardane et de la Tenora » $(O ., 558)$, « Mille regrets » $(O$., 564), «Incertitudes », «Villonelle » $(O ., 562 \&$ 604); 14 avril $1936:$ : Pas encore » $(O ., 481)$, « Les Bœufs » $(O ., 466)$, «Petit examen de conscience du dimanche 15 mai 1916 à trois heures de l'après-midi » $(O ., 511) ; 5$ juin 1937 : «Prière » ou « Pas encore » (les registres et programmes différent); 4 février 1939, Pierre Dux interprète « Ballade de la visite nocturne », Pierre Bertin «Printemps et cinématographes mêlés » $(O ., 455 ; 1461)$; 13 avril $1940^{27}: \ll 1900 »(O ., 461)$.

\& 18 mars $1922^{28}$, Salle Pasdeloup, 10 rue des Ursulines, Paris. Compagnieécole Charles Dullin : L'Atelier

«Chantage », (op. cit.) : Dullin est « M. Iklé».

\& 18 février 1922, même lieu, même compagnie :

« Entrepôt Voltaire, gros et détail, articles en tous genres » (op. cit. $)^{29}$.

๔ 23-26 mai 1921, Théâtre Michel, 38 rue des Mathurins. Spectacle organisé par Pierre Bertin ${ }^{30}$, La Femme fatale ${ }^{31}$ :

pièce en 1 acte enregistrée à la Sacem sous le titre « Le Mort vivant 》 et à la SACD sous le titre « Le Mort assassin ». Création de Pierre Bertin sous le titre «Diane de Mantoue, Femme fatale ${ }^{32}$ ». Le mensuel Montparnasse $\mathrm{du} 1^{\mathrm{er}}$ novembre donnera un extrait de la partition de Georges Monier, hauboïste belge, créée ultérieurement pour la pièce qui diffère de la musique de scène restée inconnue. Le programme comprenait également Caramel mou, shimmy pour Jazz-band de Darius Milhaud et trois autres comédies- 

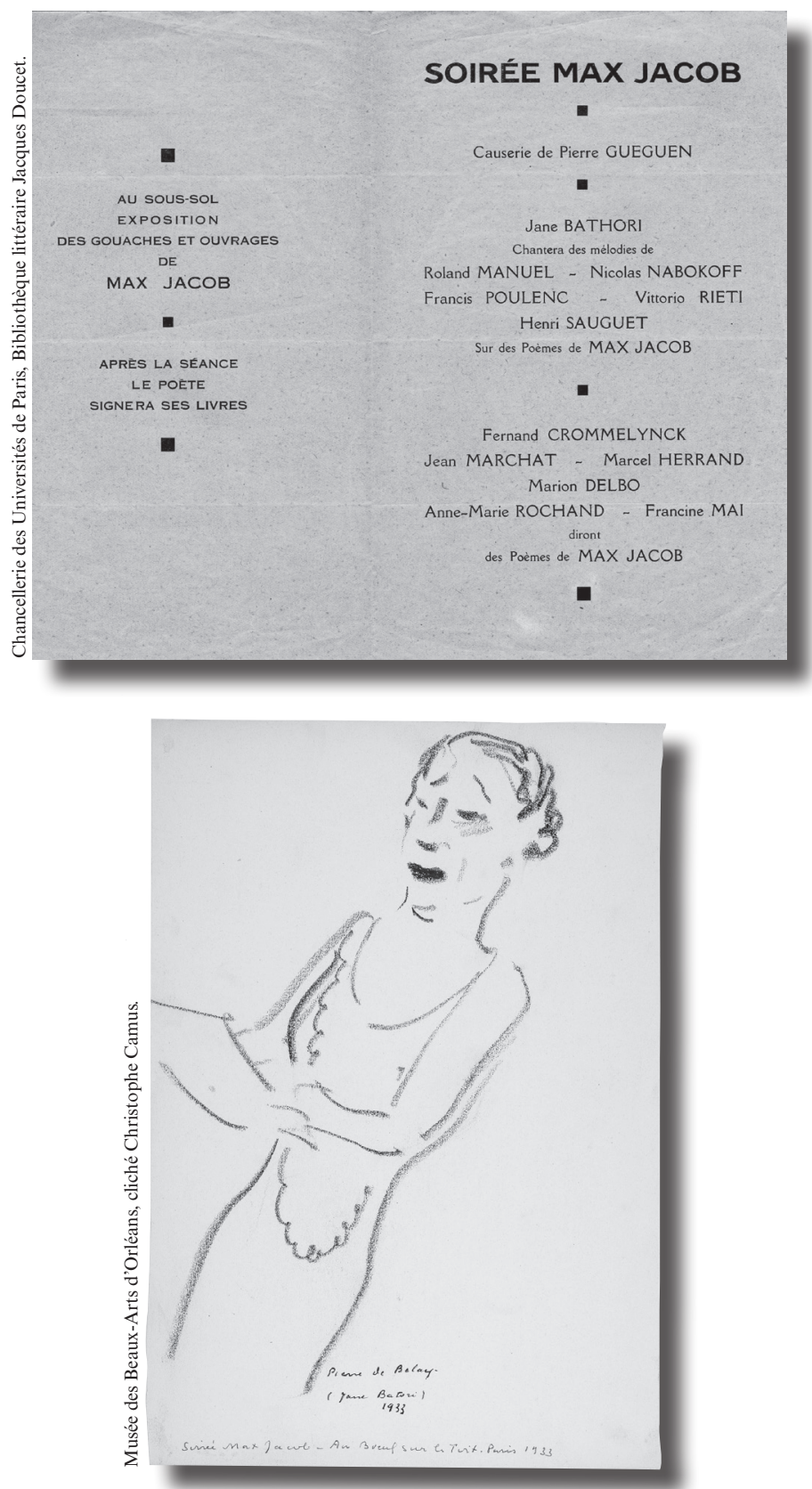

Pierre de Belay, (Jane Batori)/1933/Soirée Max Jacob au Bouf sur le toit, Paris, 1933, crayon noir sur papier. 
bouffes : Le Piège de la Méduse, comédie lyrique en un acte d'Erik Satie ${ }^{33}$; Les Pélican ${ }^{34}$, pièce en deux actes, de Raymond Radiguet, musique de Georges Auric ; Le Gendarme incompris ${ }^{35}$, pièce en deux actes de Jean Cocteau et Raymond Radiguet, musique de Francis Poulenc.

\& 8 mars 1920, Galerie Barbazanges, 109 rue du Fb. Saint-Honoré :

Ruffian toujours, truand jamais, pièce en trois actes. Scénographie de Pierre Bertin, Erik Satie compose deux volets de Musique d'ameublement joués pendant les entr'actes (voir infra, p. 327-355).

\& 24 juin 1919, 21 h 30, même lieu, Grand gala d'avant-garde organisé par Pierre Bertin à l'occasion de l'exposition des peintres Gontchorova et Larianow [sic] $1^{\text {re }}$ PARTIE : Fragment du Sacre du printemps par Marcelle Meyer et Georges Auric; « Berceuses du chat» et «Trois petites pièces pour piano» de Stravinsky par Marcelle et Germaine Meyer ; « Deux petits airs » (Mallarmé/Darius Milhaud) ; « La Vierge aux rochers » (Max Jacob/Roland-Manuel, O., 374), par Jane Bathori ; "Chapelles Romaines » de Georges Auric ; « Le Bestiaire d'Orphée » (Apollinaire/Louis Durey) ; «Idylle » d'Erik Satie ; « Heures séculaires et instantanées, Mouvements perpétuels»de Francis Poulenc ; « Jeux de plein air» musique : Germaine Tailleferre.

$2^{\mathrm{e}}$ PARTIE : «Jongleur » par M ${ }^{\text {elle }}$ Caryathis, musique : Francis Poulenc.

$3^{\mathrm{e}}$ PARTIE : Trois Nouveaux Figurants au théâtre de Nantes, nouvelle dialoguée en un acte de Max Jacob, scénographie de Pierre Bertin. Le ms est perdu, argument connu par le compte rendu de Cocteau publié dans Paris-Midi ${ }^{36}$.

Distribution $^{37}$ : L'élève : M. Gatineau ; le proviseur : M. Derland ; le censeur : M. Leflou ; le père : M. Demay ; la bonne : $\mathrm{M}^{\text {elle }}$ Ginette Leclair ; la dame du proviseur : Melle Valentine Teissier; la fille du proviseur : Melle Wilson.

Une défection de Derland a induit la reprise du rôle par Max Jacob.

\section{REPRÉSENTATIONS INCERTAINES}

e saison 1934/1935: Un amour du Titien, livret de Max Jacob, musique d'Henri Sauguet (voir infra), programmation annoncée par la compagnie Le Rideau de Paris dirigée par Marcel Herrand et Jean Marchat, Noir et blanc (18 nov. 1934) et Paris-Soir (24 nov. 1934) ${ }^{38}$.

« saison 1930/1931: Les Impôts/Maucamélia, sketch remanié par Dullin à partir de l'opérette de Cliquet-Pleyel et Max Jacob, mai-juin 1930, ms perdu ${ }^{39}$. Le 
relevé comptable de la Société des Auteurs donne la date du 26 mai 1930 sans autres mentions. Est-ce Le Kleptomane (voir supra)?

\& saison 1924/1925: Le Terrain Bouchaballe, comédie en 3 actes, Compagnie Le Pou qui grimpe (Joseph Quesnel dir.), Coutance ou Saint-Lô ${ }^{40}$.

\section{MAX JACOB DÉCORATEUR DE THÉÂTRE : PATRONAGE DE SAINT-BENOÎT}

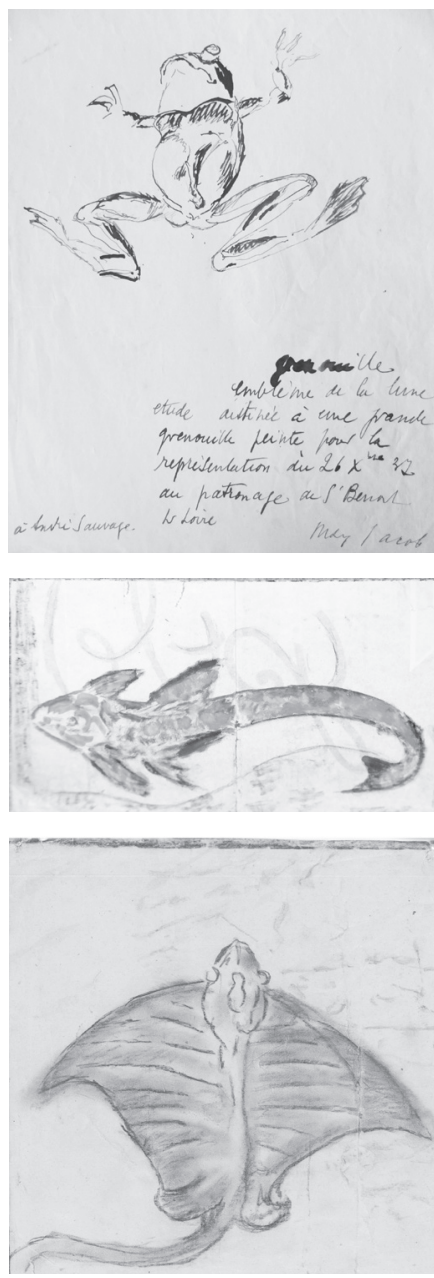

\& Étude pour grenouille peinte, encre de chine, dédicace : «Grenouille/emblème de la lune/étude destinée à une grande/grenouille peinte pour la/représentation du $26 \mathrm{X}^{\text {bre }}$ 37/au patronage de St-Benoît/sur-Loire/À André Sauvage/ Max Jacob », coll. André Sauvage/Max Jacob, MO.

\& Rayon des aquariums, gouache et crayon gras avec rehauts de crayon noir au revers, sd, 495 x 253. Dessin réalisé au revers d'un papier d'emballage bleu foncé avec arabesques dont les contours sont repris sommairement au verso (Communauté monastique de St-Benoît-sur-Loire).

\& La Raie, crayons gras de couleur et mine graphite, sur papier d'emballage, sd., 495 x 253 (Communauté monastique de St-Benoît-sur-Loire).

Le Mont-Blanc vu d'une fenêtre, peinture à l'huile, 2 m., lettre inédite à Roger Toulouse, vendredi 30 [décembre 1938]. «Petit décor qui est un four noir » (à Alain Messiaen, 16 janvier 1939, lettre inédite, MO).

\section{REPRÉSENTATIONS POSTHUMES : COMPAGNIES ET THÉÂTRES SUBVENTIONNÉS}

\& 14 avril-4 mai 1986, Théâtre Daniel Sorano-CDN Toulouse $^{41}$, Compagnie Grenier de Toulouse, 22 mai 15 juin 1986, reprise au Théâtre national de Chaillot, salle Gémier. 
Le Terrain Bouchaballe, mise en scène de Jacques Rosner, décors et costumes de Max Schoëndorff, son d'André Serré, lumières Bruno Boyer, assistante à la mise en scène Nicole Rosner. Distribution : Monique Tarbès, Maurice Chevit, Jean Bousquet, Michel Oster, Pierre Nougaro.

\& 10-18 mars 1984, Quimper, Semaine Max Jacob, Le Terrain Bouchaballe, Compagnie Les Tréteaux éclatés, mise en scène de Jean-Yves Crochemore.

^ Comédie-Française 1960-2014 : Matinées Poétiques, 7 séquences : pour chacune quelques poèmes de Max Jacob.

\& 22 décembre 1945, Théâtre de Quimper, Soirée Hommage à Max Jacob par les comédiens de la Radiodiffusion française.

\& Le Divorce de la duchesse de Prazzel et Ne coupez pas Mademoiselle. Soirée organisée par Alain Trutat et Jean Lescure.

\section{TRADUCTIONS}

e En italien

JACOB Max, L'Amoureux de la baronne/L'Amoroso della baronessa, trad. et annotations par Andrea Bedeschi, In forma di parole $\mathrm{n}^{\circ} 2,1996$ : Poetare e pensare, Bologna, 1996, p. 84-97.

$\propto$ En russe

JACOB Max, Le Roi de Béotie suivi de Filibuth ou la montre en or, trad. d'Alla Smirnova, Moscou : éd. Sankt, 2003.

\section{CORRESPONDANCES ADRESSÉES À DES ACTEURS, COMÉDIENS, DIRECTEURS DE THÉÂTRE...}

\& GOLDBLATT Charles: JACOB Max, L'Amitié, lettres à Charles Goldblatt, corr. établie et présentée par André Roumieux, Bègles : Le Castor Astral, 1994.

« HERTZ Henri : JACOB Max, "Lettres à Henri Hertz, 1923-1926 ", corr. établie et présentée par René Plantier, CES n 3, 1980, p. 71-82. 
« LUGNÉ Aurélien-Marie, dit Lugné-Poe, dans FRAYSSE Jean : Max Jacob and "les feux de Paris », unpublished letters from Max Jacob to Jean Fraysse, correspondance présentée par Neal Oxenhandler, Berkeley/Los Angeles : University of California Press, coll. University of California Publications in Modern Philology, vol. 35, n 4, 1964, p. 254-255.

\& RÉMY Tristan : JACOB Max, Fragments de pensées et de vie littéraire dévoilés dans quelques lettres de Max Jacob à Tristan Rémy, 1922-1923, édités et commentés par Alain Segal, Tusson : Du Lérot éditeur, 2014.

@ ROLAND-MANUEL: JACOB Max, "Agréez, Monsieur et cher collaborateur, l'assurance de ma considération très distinguée. " Lettres à Roland-Manuel (1917-1929), corr. revue et augmentée, éd. critique de Patricia Sustrac, voir la publication dans ce numéro.

\& SALACROU Armand : JACOB Max, Lettres aux Salacrou août 1923 - janvier 1926 avec un portrait de Max Jacob par André Baudin, Gallimard, 1957.

« SALOU Louis : JACOB Max, "Je t'embrasse, sensible et donc fidèle..." Lettres inédites à Louis Salou (1930 ? - 1937), éd. critique de Patricia Sustrac, dans $C M J 19 / 20$, voir la publication dans ce numéro.

\section{ÉTUDES ET ESSAIS}

ANDREU Pierre, Vie et mort de Max Jacob, La Table ronde, 1982, p. 99-10, 205-230.

\& AURENCHE Jean, Entretiens avec Anne et Alain Riou, Lyon, Institut Lumière/Actes sud, 1992.

œ BERNAC Pierre, Francis Poulenc et ses mélodies, Buchet Chastel, coll. Libello, 2014, p. 163-173.

\& BERTIN Pierre, Le Théâtre et (est) ma vie, propos recueillis par Robert Tatry, Le Bélier, 1971. 
\& BOUDIN-LESTIENNE Stéphane, MARE Alexandre, Charles et MarieLaure de Noailles, mécènes $d u X X^{e}$ siècle, Villa Noailles centre d'art \& Bernard Chauveau édition, 2018, p. 56-59; 240-265 ; 266-269.

^ BRASSEUR Pierre, Ma vie en vrac, Calmann-Lévy, 1972, p. 134-138. - rééd. Ramsay Poche cinéma, 1986 (même pagination).

« CHIMÈNES Myriam, Mécènes et musiciens, du salon au concert à Paris sous la III République, Arthème Fayard, 2004 [nombreuses réf. aux musiciens de Max Jacob].

\& CORVIN Michel, Le Théâtre de recherche entre les deux guerres. Le laboratoire Art et Action, Lausanne : L'Âge d'homme/La Cité, coll. Théâtre des années vingt, 1976 [réf. à Bertin et Jacob, extraits de lettres à Claude Autant-Lara].

« D'AUBARÈDE Gabriel, De mémoire oubliée, souvenirs littéraires, dessins de Roger Wild, éd. établie et présentée par Étienne de Montéty, La Table ronde, 2004, p. 146-154 [contribution de Jacob aux Cahiers du film et Noctambules].

\& DAUFRESNE Jean-Claude, Fêtes à Paris au XXe siècle. Architectures éphémères de 1919 à 1989, Sprimont : éd. Mardaga, 2001 [bals et soirées privées des années 1928-1930 auxquels Max Jacob a été invité, musiciens et œuvres de Max Jacob].

@ DUCHESNEAU Michel, L'Avant-garde musicale à Paris de 1871 à 1939, Mardaga, 1997.

« DICKOW Alexander, Jacob et le cinéma, Nvlles éd. J.- Michel Place, coll. Le cinéma des poètes, $2017^{42}$.

« ÉMIÉ Louis, Dialogues avec Max Jacob, Corrêa-Buchet-Chastel, coll. Mises au point, 1954.-rééd. Bordeaux : Le Festin, 1994, p. 57-59, 137-139.

\& GABORY Georges, \& Compagnie, Apollinaire, Max Jacob, Gide, Malraux, éd. J.- Michel Place, 1988, p. 21-51.

« GUÉRIN Jeanyves, Le théâtre en France de 1914 à 1950, Honoré Champion, coll. Dictionnaires et références, 2007. 
\& GUIETTE Robert, La Vie de Max Jacob, A. G. Nizet, 1976, p. 124-127, 140143, 147-151.

\& HELL Henri, Francis Poulenc : musicien français, Fayard, 1978, p. 80, 101-116, 195, 291, 325, 340 [Le Bal masqué et autres adaptations des poèmes de Jacob].

« HELL Henri, Arthur Honegger. Un musicien dans la cité des hommes, Fayard/ Sacem, 1992 [contient : La Mort de sainte Alméenne de Max Jacob, p. 546, voir aussi infra].

\& HONEGGER Arthur, Lettres à ses parents, 1914-1922, préfacées et annotées par Harry Halbreich, Genève : éd. Papillons, coll. $7^{\mathrm{e}}$ note, 2005, p. 129-130, $172,175,184,202,228,325$.

\& LACHGAR Lina, Max Jacob, éd. Veyrier, coll. Les Plumes du temps, 1981, p. 50-53 [témoignage de Pierre Bertin].

\& LACOMBE Hervé, Francis Poulenc, Fayard, 2013.

œ MILHAUD Darius, Notes sur la musique, essais et chroniques, textes réunis et présentés par Jérémy Drake, Flammarion, coll. Harmoniques, 1982, p. 60 sq.

@ MOUSLI Béatrice, Max Jacob, Flammarion, coll. Grandes biographies, p. 186-192, 217-219, 348-350, 391-393.

\& OLIVIER Fernande, Picasso et ses amis, Pygmalion, 2001 [chapitre consacré à Max Jacob : évocations de ses talents musicaux, dramaturgiques et dons d'imitation].

\& PÉRARD Joseph, Max Jacob l'universel, études, inédits, Colmar : Alsatia, 1974, p. 65-69.

@ PEYRE André, Max Jacob au quotidien, José Millas-Martin éditeur, 1976, p. 45-57.

\& POULENC Francis, Journal de mes mélodies, Grasset, 1964, p. 113-117, 143144, 152, 167-171, 176-179, 191, 195, 220-221, 243, 258, 263, 268, 273. 
\& POULENC Francis, Correspondance 1910-1963, réunie, choisie, présentée et annotée par Myriam Chimènes, Fayard, 1994.

\& RIM Carlo, Le Grenier d'Arlequin, Denoël, 1981 [voir l'index : théâtre, conférences, contributions à Jazz, propos de Max Jacob et correspondances].

SACHS Maurice, Au temps du Bœuf sur le Toit, Grasset, 1987.

\& SALACROU Armand, Dans la salle des pas perdus, Gallimard, T. 1 : C'était écrit, 1974 ; T. 2 : Les Amours, 1976.

\& SAUGUET Henri, La Musique, ma vie, Librairie Séguier/Archambaud, 1990 rééd. 2001, p. 159, 163-165, 216, 230, 253-254, 257, 277-280, 289-291, 359-361 [couverture de la partition des Pénitents et facsimile de lettres à Henri Sauguet.]

\& SCHMITT-KUMMERLEE Philippe, Max Jacob au grand Quartier Général Nollet, un nouveau Bateau-Lavoir, éd. Al Manar, coll. Approches et rencontres, 2007.

œ YAKI Paul, Le Montmartre de nos vingt ans, Taillandier, 1949, p. 114-129.

\section{THÈSES ET MÉMOIRES ${ }^{43}$}

« BASSET Florence, "La Technique de la comptine chez Max Jacob », mémoire de Maîtrise de Lettres Modernes sous la direction de Mme Veyrenc: Université de Reims, 1987.

« SAINT-THOMAS Grâce-Mary, « Les Éléments du comique dans l'œuvre de Max Jacob », doctorat de littérature française : Faculté des Lettres de Paris-Sorbonne, 196844. [les p. 133-137, 144-146, 216-226, 267-269 sont consacrées au théâtre].

\section{REVUES, BULLETINS, OUVRAGES COLLECTIFS}

\& Les Cahiers Max Jacob, n 19/20, Barcelone : Les Amis de Max Jacob, 2019 : so AMBROISE Guillaume, « Medrano et Max Jacob », p. 225-227 ;

so BRUNEL Pierre, "Sur Un amour du Titien, "opérette inspirée en 5 actes et 4 tableaux" de Max Jacob et Henri Sauguet », p. 357-365; 
so COLLIN Florence, "Le Bal masqué, cantate profane de Francis Poulenc », p. 107-129;

sos DICKOW Alexander, "Trouver l'intrus : le théâtre dans Le Roi de Béotie », p. 263-276;

so FARGETON Pierre, "Le swing ou "la sensation de l'irremplaçable". Autour d'une interview inédite d'Hugues Panassié par Max Jacob (1936) », p. 179-195;

so GANDEBEUF Jean-Pierre, et JACQUIER-ROUX Jean-Louis, " Max Jacob et Erik Satie : amis de cœur, amis de cour? », p. 476-482 ;

so JACOB Max, «Une histoire de pianos », nouvelle inédite présentée par Patricia Sustrac, p. 210-222; JACOB Max-ROLAND-MANUEL, «Agréez, Monsieur et cher collaborateur, l'assurance de ma considération très distinguée... », correspondance (1917-1929) revue et augmentée présentée par Patricia Sustrac, p. 31-102 ; JACOB Max-vicomte et vicomtesse de NOAILLES, " Être des vôtres est une bénédiction véritable... », lettres inédites, correspondance croisée (1929-1934) présentées et annotées par Patricia Sustrac en collaboration avec Stéphane Boudin-Lestienne et Alexandre Mare, p. 131-148; "Chansons », p. 149-162 ; « Jazz, interview inédite d'Hugues Panassié suivi du poème « Louis Armstrong 》 (Jazz hot, 1936) », textes établis par Patricia Sustrac, p. 168-178; « À Propos de théâtre ", texte d'esthétique présenté par Patricia Sustrac, p. 254-262 ; Les Pétroles du Béloutchistan, Ruffian toujours, truand jamais, comédies présentées par Patricia Sustrac, avant-dire de Jean-Claude Penchenat, p. 289-355 ; JACOB Max-SALOU Louis, " "Je t'embrasse, sensible et donc fidèle...", lettres inédites (1928-1937)», p. 366-388;

so JACOB Pascal, « Ombres et miroitements », p. 229-240;

ss LE GUILLOU Philippe, «Les Chants de Morven », p. 163-167;

so MALRIEU, Jean, «La Vie de château. Max Jacob chez Hugues Panassié », texte commenté par Pierre Fargeton. Témoignage suivi d'une lettre inédite de Max Jacob à Jean Malrieu, p. 197-209;

so PONTIER Jean-Marc, « Max Jacob croque le cirque », p. 241-251;

sos SUSTRAC Patricia, "Max Jacob et les arts de la scène », p. 10-29, « Max Jacob et les arts de la scène : Liber amicorum suivi de trois lettres inédites de Charles Trenet et Johnny Hess à Max Jacob, 1934 », p. 390-435; « Max Jacob et les arts de la scène. Orientations bibliographiques », p. 437-474;

so VAN ROGER-ANDREUCCI Christine, « Le Siège de Jérusalem, grande tentation dramatique de Max Jacob », p. 277-288;

\&s Cahier hors-texte couleur encarté entre les pages 240 et 241. 
\& Lettres \& Mots, bulletin semestriel de l'Association des Amis de Max Jacob :

$\operatorname{son} \mathrm{n}^{\circ} 11,2^{\mathrm{e}}$ semestre 2012 : SUSTRAC Patricia : "Max Jacob aimait-il le cinéma? », p. 1-3.

\&s n 6, sept. 2007 : MARX René, «La critique [sur Monsieur Max] », p. 7 ; SUSTRAC Patricia : « Max Jacob, le film !» p. 1-2, «Monsieur Max : les auteurs », p. 6-7, « Monsieur Max : une distribution d'exception », p. 8.

« Hors- Jeu, hommage à Max Jacob (tome 2) : Hors-Jeux éd., juin 2002 :

œ HENRY Hélène, « Le Roi de Béotie (une œuvre confluence)», p. 5-14.

\& Le Cri d'os: La Lucarne Ovale, 1994, n 7

so DESPERT Jehan, « Max Jacob et la musique », p. 33-37.

\& Cahiers du Centre de recherches Max Jacob (CES), n 9, 1987 :

so HENRY Hélène, "Chronologie du théâtre de Max Jacob », p. 45-49, « Du dialogue à la farce : deux inédits de Max Jacob », p. 50-51, « Bouchaballe de ma jeunesse ou la grande tentation terrestre de Max Jacob», p. 57-60; JACOB Max, «Paris Province, dialogues », p. 52-54, " Petits dialogues : peintre et marchand », p. 55-56; Dossier de presse du Terrain Bouchaballe, p. 61-84.

« Théâtre à Toulouse, $\mathrm{n}^{\circ}$ 3, avril-juin 1986 :

so HENRY Hélène, "Le sous-terrain de l'affaire Couchouren ", p. 4-8, « Masque Jacob», p. 10 ; JACOB Max, «"Petits dialogues" : deux saynètes inédites de Max Jacob, "Peintre et marchand" », p. 11.

\& L'Avant-scène Théâtre, ${ }^{\circ}$ 798, 15 nov. 1986 :

so HENRY Hélène, "Un théâtre pour Le Terrain », p. 39-41, " Max Jacob, comédien de génie », p. 42-43, « Du dialogue à la farce », p. 43, « Liste connue du théâtre de Max Jacob », p. 60 ; JACOB Max «Le Terrain Bouchaballe » (version scénique), p. 3-35 ; «Deux inédits de Max Jacob : "Paris-Province, dialogues", "Petits dialogues, peintre et marchand" ", "Le Journal de mode ou les ressources de Florimond », p. 44-59.

\& CES, n 5,1983 :

so HENRY Hélène, "Bouchaballe "UN" ou la comédie retrouvée », p. 5-12, "Les surprises d'une topographie jacobienne (suite du n ${ }^{\circ}$ ) », p. 31-41; « Document : main basse sur la ville ou KEMPER Cancans », p. 83-87. 
@ Revue des Lettres Modernes, série « Max Jacob» (2), dir. Jean de Palacio : Minard, n 474-478, 1976 (6) :

so PELLETIER Christian «Le Terrain Bouchaballe : de la comédie au roman », p. 35-56; THOMAS Henri « La comédie Bouchaballe», p. 19-22.

\& Créer, n² 23, février-avril 1976: Max Jacob. Max Jacob 1876 (mort à Drancy 1944) - 1976 : centième anniversaire de sa naissance, Rochechouart : Centre littéraire et artistique de Rochechouart, 1976 :

so BERTIN Pierre, "Max Jacob avait un pied dans tous les arts... », p. 28, «Madame la Dauphine [partition], p. 29 ; DOUAI Jacques, « Jacques Douai chante Max Jacob » [partition], p. 57 ; MESSIAEN Alain, « Max Jacob et la musique », p. 60-63 ; SAUGUET Henri, « Une journée avec Max Jacob », p. 30-31.

\& [Collectif] Pour en revenir à Max Jacob, textes et dessins inédits de Max Jacob: José Millas éditeur, 1969 :

sos AUCLAIR Raoul, "Le Siège de Jérusalem », p. 67-71 ; FOLLAIN Jean, «Max, rue Nollet », p. 25-29.

europe, $\mathrm{n}^{\circ}$ 348-349, avril-mai, 1958 :

so FILLACIER Sylvette, « Max Jacob mon ami », p. 97-101 ; GARNIER François, " Max Jacob et le théâtre », p. 37-46 ; SAUGUET Henri, « Une journée de Max Jacob à l'Hôtel Nollet en 1932», p. 26-32.

^ Les Cahiers Max Jacob, Théâtre (I), Un amour du Titien, La Police napolitaine: Les Amis de Max Jacob, 1953, voir supra.

๑ La Boîte à clous, [n hommage à Max Jacob], $\mathrm{n}^{\circ}$ 10, février 1951, np :

s SAUGUET Henri, "Quand j'écrivais une opérette avec Max Jacob » suivi d'un extrait d'Un amour du Titien (acte I, scène II).

\& Le Mail, $\mathrm{n}^{\circ}$ 5, avril 1928 :

so JACOB Max « Le Veuf, fragment », p. 252-257 ; LE DANTEC Gérard, « Max Jacob et la muse celtique », p. 264-266. 


\section{ACTES DE COLLOQUE, JOURNÉES D'ÉTUDE, CATALOGUES D'EXPOSITIONS}

\& Visage de Roland-Manue ${ }^{45}$, journée d'étude du 17 novembre 2016, Conservatoire national supérieur de musique et de danse de Paris : CNSMD/ Les Amis de Maurice Ravel.

so ESCANDE Dominique "Isabelle et Pantalon »; SUSTRAC Patricia : « Max Jacob-Roland-Manuel, une correspondance poétique et musicale partagée. »

\& Max Jacob et la création suivi des inédits de Max Jacob Paris province et Le Journal des modes ou les ressources de Florimond. Actes du colloque d'Orléans, 4-5 mars 1994 organisé par la ville et l'Université d'Orléans, éd. J.-Michel Place, coll. Surfaces, 2000 :

so HENRY Hélène, "Tentatives et tentations théâtrales de Max Jacob », p. 85-89, "Chronologie et bibliographie du théâtre de Max Jacob », p. 9094 ; JACOB Max, « Paris-Province, dialogues », « Petit dialogue : peintre et marchand », « Le Journal des Modes ou les ressources de Florimond », p. $97-132$.

\& Max Jacob à la Confluence, actes du colloque de Quimper, 21-23 oct. 1994, Quimper : Bibliothèque Municipale de Quimper/Université de Bretagne occidentale- Faculté des Lettres Victor Segalen, Brest, 2000 :

so HENRY Hélène, « Le Roi de Béotie, une œuvre confluence », p. 231-242.

\& Max Jacob poète et romancier, actes du colloque du CRPC/Université de Pau, 25-28 mai 1994 avec des lettres inédites de Max Jacob à Valéry Larbaud et à Jean Cocteau, textes réunis par Christine Van Rogger-Andreucci, $n^{\circ} 4$, avril 1995 :

So ALBERT Christiane, "La théâtralité dans Le Roi de Béotie », p. 89-96 ; RÉAL Éléna, « Les jeux de l'ironie dans Le Roi de Béotie », p. 83-89.

\& L'Archange foudroyé, Musée des Beaux-arts d'Orléans, 23 avril-31 juillet 1994, Orléans : Musée des Beaux-arts d'Orléans, 1994 :

so CLUZEL Raphaël, " Les Années Nollet », p. 68-69, "Les Musiciens de Max Jacob », p. 70-74; SAUGUET Henri « Max Jacob et les musiciens », p. 65-67.

so VOLTA Ornella, « Max Jacob et Erik Satie », p. 77-79. 
\& Qui (ne) connaît (pas) MAX JACOB ? Actes du colloque de l'Université du $3^{\mathrm{e}}$ âge et pour tous, Lorient : Institut culturel de Bretagne, 1987 :

so HENRY Hélène, "Présence et tentation du théâtre dans l'œuvre de Max Jacob », p. 57-81, « Liste connue du théâtre de Max Jacob », p. 78-79.

๔ CES, Actes du colloque de Saint-Étienne, 27-30 sept. 1983, nº 7, 1985 :

sos HENRY Hélène, « La première "nouvelle de Guichin" en 1910 », p. 18-29.

\section{ARTICLES}

« CHIMÈNES Myriam, "Francis Poulenc et les poètes », XX $X^{e}$ siècle, revue d'histoire, vol. 49, n 1, janv.-mars 1996, p. 146-14846.

« DARRAS Jacques, « Max Jacob et la musique », MJ Mag, janv. 1994, p. 37.

« GOURVIL Francis, "Max Jacob collecteur de chansons bretonnes ", Nouvelle revue de Bretagne, mai-juin 1949, p. 228-231.

\& HENRY Hélène, «Une revue quimpéroise en 1910 par Max Jacob et Pierre Allier », Les Cahiers de l'Iroise, $\mathrm{n}^{\circ} 1$ ( ${ }^{\text {vlle }}$ série), janvier-mars $1962,9^{\mathrm{e}}$ année, p. 7-12.

so HENRY Hélène, "Le Terrain Bouchaballe et Quimper », Les Cahiers de l'Iroise, $\mathrm{n}^{\circ} 1$ ( $\mathrm{n}^{\mathrm{vlle}}$ série), janvier-mars 1985, 32 année, p. 31-38.

@ MAGNENAT Nadedja, "Max Jacob et le cinématographe », CMJ 15/16, p. 61-74.

@ PELLETIER Christian, "Le Terrain Bouchaballe, comédie inédite de Max Jacob », Les Cahiers de l'Iroise, avril-juin 1972, $19^{\mathrm{e}}$ année, $\mathrm{n}^{\circ} 2$ (n ${ }^{\text {vlle }}$ série), p. $96-99$.

« SAUGUET Henri, « Max Jacob et la musique », Revue musicale, janvier 1952, p. 151-154 suivi de « Un amour du Titien, opérette inspirée en 5 actes et 4 tableaux, musique par Henri Sauguet » (extrait), p. 155-159.

œ SUSTRAC Patricia, "Vous allez donc au cinématographe ? », CMJ 7, p. 124129. 


\section{LES COLLECTIONS PUBLIQUES}

\section{À LA BIBLIOTHÈQUE NATIONALE DE FRANCE}

DÉPt. DEs ArTs du SPECTACle

« Fds Louis Jouvet:

so Max Jacob, Journal des modes ou les ressources de Florimond, ms autog. remis à Louis Jouvet le 28 avril 1931. Ms à l'encre noire numéroté par l'auteur h. d. de 1 à 47, recto (ff. 1 à 5 : prologue;f. 6 : décor;ff. 7 à 41 : texte) ms très au net monté sur onglet et sous reliure bradel, titre et nom de l'auteur en lettres d'or sur la tranche. Aucunes marginalia.

\section{Dépt. des Manuscrits : Fds Max Jacob-Collection Gompel-Netter}

« Épreuves corrigées

so Le Phanérogame

Épreuves corrigées datées d'avril 1905. En page d'avant-titre, un envoi (à l'imprimeur Levé ?) : «Si précieux que soit/ce petit paquet/il ne l'est pas autant/qu'un peu d'amitié/de vous/Max Jacob ». En faux titre, mention de sous-titre « Roman paradoxal/mêlé de couplets et de cavalcades » biffé au profit du sous-titre retenu pour l'édition. Nombreuses annotations destinées à l'imprimeur (épreuves manquantes, pages sautées, indications des espaces et des blancs). Inscriptions au crayon graphite en bas de quelques ff. par les protes Gourmel et Formel.

\section{MANuscrits aUtographes}

œ Cinéma

7 ff., 275 x 213, écrits à l'encre noire au ${ }^{\circ}$ monté sur onglets suivis de la revue Heim dans laquelle il est reproduit p. 16-17. Texte illustré d'un dessin de Marc Réal représentant un plateau de cinéma et d'un cul-de-lampe du même.

\section{so Le Roi de Béotie}

$95 \mathrm{ff}$., formats divers, encre bleue, violette et crayon graphite, ms très raturé et corrigé par l'auteur écrit au $\mathrm{r}^{\circ}$ sauf quelques annotations marginales au $\mathrm{v}^{\circ}$ : «Par le gros bout de la lorgnette »: 42 ff. ; " Nuits d'hôpital et l'aurore » : $53 \mathrm{ff}$. Transcription dactylographiée corrigée par Didier Gompel : $3 \mathrm{ff}$. manquants : " Chantage » et « Mémoires du Père Vaubois », ce dernier conservé à la MO.

\& Jazz, voir la publication dans le présent numéro. 
\& À Propos de théâtre, voir la publication dans le présent numéro.

\& La Femme fatale

$25 \mathrm{ff}$. dont 21 de Max Jacob et 4 de Pierre Bertin. Ms à l'encre noire, bleue et violette (ff. 19 à 25), crayon graphite et de couleur. Indications de mise en scène au crayon graphite noir et rouge. Papier ligné utilisé en format paysage et portrait en $r^{\circ}$. Paragraphes et répliques raturés à l'encre ou au crayon graphite. $V^{\circ}$ f. 11 dessins de Max Jacob, croquis d'une scène. $V^{\circ}$ f. 13 adresse $\mathrm{du}$ « docteur Martinet : 24 rue Chaptal, professeur, consultations ». $\mathrm{V}^{\circ}$ f. 14 , note aut. : "Vendu tes cadres/Je ne suis pas loin/je reviens/attends-moi »; $\mathrm{v}^{\circ}$ f. 25 : une esquisse de dessin à l'encre violette. Est jointe une enveloppe contenant trois ms aut. : répliques de personnages de la pièce au $\mathrm{v}^{\circ}$ de pages d'une revue paroissiale de St-Benoît-sur-Loire.

\& La Sainte Hermandade

$10 \mathrm{ff} .24$ x33 numérotés par l'auteur h. d. de 1 à $10,1 \mathrm{f} .13 \times 22 \mathrm{~cm}$ papier ligné $\mathrm{r}^{\circ}$ et $v^{\circ}$ résumé rapide de présentation du deuxième acte et dialogues de la scène $\mathrm{I}$.

\& L'Amoureux de la baronne

4 ff., 32 x $24 \mathrm{~cm}, \mathrm{r}^{\circ}$, à l'encre noire et une copie dactylographiée.

$\propto$ L'Enfant de Lolotte L $^{47}$

$25 \mathrm{ff}$ : : plan $2 \mathrm{ff}$., notes préparatoires : $3 \mathrm{ff}$., scénario $1^{\mathrm{er}}$ acte : $23 \mathrm{ff}$. Transcription dactylographiée $17 \mathrm{ff}$.

\& La Fée des écoliers ${ }^{48}$

$25 \mathrm{ff}$. au crayon graphite écrits au $\mathrm{r}^{\circ}$ datés de 1922.

« Les Pétroles du Béloutchistan, voir la publication dans ce numéro.

\& Ruffian toujours, truand jamais, voir la publication dans ce numéro.

« La Mort de Sainte Alméenne, voir supra.

\& Chansons, voir la publication dans ce numéro.

\& Partitions anthumes et posthumes (copies ou originales)

Paul Arma, Georges Auric, Henri Bordes, Robert Caby, André Jolivet, René Leibowitz, Roland-Manuel, Francis Poulenc, Vittorio Rieti, Henri Sauguet, Virgil Thomson. 


\section{\& Correspondances}

Lettres de Max Jacob à : Jane Bertrand-Ager, Jean Aurenche, Georges Auric, Pierre Bertin, Sylvette Fillacier, Franz Hellens, Marcel Herrand, Henri Hertz, Roland-Manuel, Armand Salacrou.

\section{DéPt. De la Musique}

« Fds contenant des lettres de Max Jacob adressées à : Jane Bathori, André Caplet, Francis Poulenc, Roland-Manuel, Henri Sauguet.

\section{BIBLIOTHÈQUE LITTÉRAIRE JACQUES DOUCET}

\section{MANUSCRITS AUTOGRAPHES}

« L'Acteur de cinéma ivre ${ }^{49}$

4 ff. à l'encre noire. En marge g. $\mathrm{r}^{\circ}$ de biais au crayon graphite : "Conte de film », en marge d. $r^{\circ}$ au crayon gras rouge : « Film ».

\section{« Histoire de Don Juan}

Ms ayant servi à l'impression de l'article paru dans Nord-Sud, 15 mars 1917, $\mathrm{n}^{\circ}$ 1, p. 13-15 ; 4 ff. à l'encre noire sur papier ligné au $\mathrm{r}^{\circ}$, dim. $21 \times 27$. Indications de saisies des protes au crayon graphite. Nombreuses disparités avec la publication homonyme en volume (voir supra).

\section{DESSINS}

« DANTZIG Héram ou Hiram, Max Jacob au piano vers 1895 , dessin au fusain.

\& GODIEN Adrien, Max Jacob au piano, vers 1898, fusain. Légende aut. : « Moi avec une barbe/au piano à Quimper/vers 1898/par un peintre lyonnais nommé Godien. »

\section{CORRESPONDANCES}

Lettres reçues de George Auric, Georges Bernanos, Charles Dullin ${ }^{50}$, Armand Salacrou.

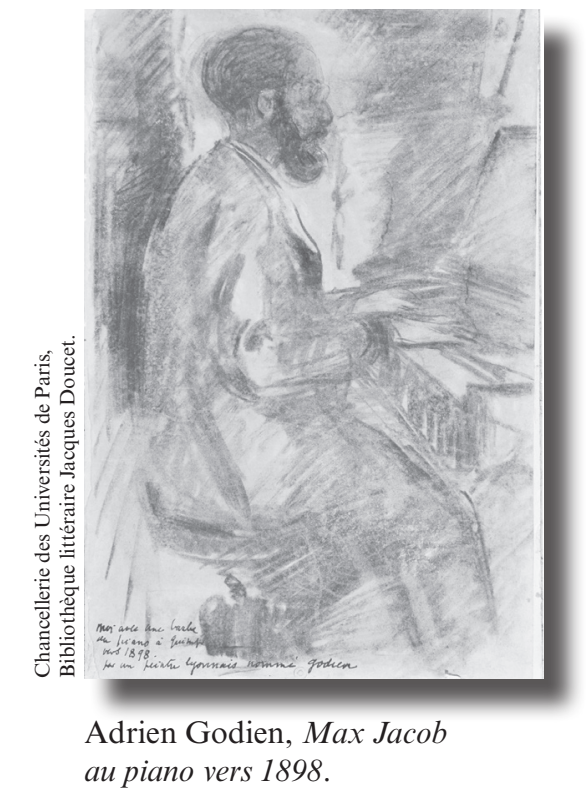




\section{MUSÉE NATIONAL D'ART MODERNE, CENTRE POMPIDOU}

\section{Collections GRaphiQues}

\& Le Pardon, scène de comédie, 1930, aquarelle et gouache sur papier.

\& À L'Eldorado, 1930, aquarelle gouache et encre sur papier.

\& À L'Opéra, 1930, gouache sur carton.

\section{MÉDIATHÈQUE DES URSULINES, QUIMPER}

\section{ÉPREUVES D'IMPRIMERIE}

\& Les CEuvres burlesques et mystiques de Frère Matorel mort au couvent de Barcelone

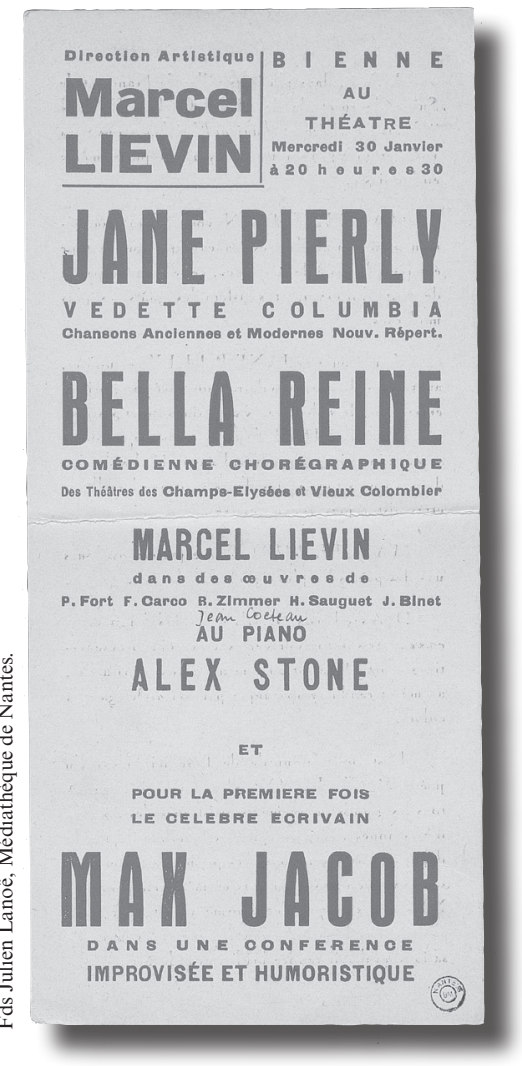

Tournée Liévin, Nantes, 30 janvier 1935.
6 ff., épreuves d'impressions dont la couverture, annotations manuscrites à la plume et au crayon graphite, en couverture : " [erreurs ?!] faites attention !/tous ces poèmes appartiennent à/ Kahnweiler et ne peuvent être publiés./Tout ceci a été publié en 1909 ou 10/et écrit vers $1900 »$. En faux-titre : «Attention !/poèmes appartenant/à Kahnweiler et ne pouvant/être publiés.

\section{ManUSCRits aUtOGRaPhes et TAPUSCRIT}

\& Pierre Allier et Max Jacob (tapuscrit) Kemper

Cancans $^{51}$, pièce en 4 actes, août 1910, $96 \mathrm{ff}$.

« «Une histoire de pianos ", voir la publication dans ce numéro.

\section{ARTICLES DE PRESSE RASSEMBLÉS PAR L'AUTEUR OFFERTS À LA BIBLIOTHÈQUE ${ }^{52}$ \\ « «Max Jacob librettiste » : 13 articles de 1935 à 1936,}

« « Max Jacob conférencier»: 21 articles relatifs à la tournée de $1935^{53}$ avec les comédiens et danseurs Jane Pierly, Bella Reine, Marcel Liévin, \& 3 articles relatifs à la revue Mouvement (voir supra),

$\propto 24$ articles relatifs au spectacle des Noctambules. 


\section{MUSÉE DES BEAUX-ARTS DE QUIMPER}

CEUVRES GRAPHIQUES, CORRESPONDANCES

Voir le catalogue en ligne des œuvres du Musée.

\section{Archives municipales de Quimper}

\& ALLIER Pierre, JACOB Max, Kemper-Cancans, voir supra.

\& Fds Hélène Henry. Le fonds contient des matériaux d'étude, nous indiquons les références susceptibles de contenir des éléments complémentaires aux articles cités supra.

so «Kemper - Cancans "en l'état" : la revue scène à scène »

so « Kemper Guichen de Pierre Allier à Max Jacob »

\$ « Une espèce de revue dans un théâtre forain (Max Jacob) = Kemper Cancans, été $1910 »$

so La troupe Bénévent (théâtre-cirque et son répertoire à Quimper en 1903 et 1910)

\$) «Max Jacob sur son terrain » suivi de «Bouchabal masqué »

\&s « 1903 Quimper : Les attaques de L'Action Libérale contre la salle des fêtes et le pont en ciment armé »

ş «Une description élogieuse de la salle des fêtes à Quimper »

so « L'inauguration officielle du théâtre de Quimper, 19 février 1904 »

\&) «Bouchabal masqué, une littérature "carnavalisée" "; " Musiques sur Le Terrain : étude : Bouchaballe»

œ) «Notes sur Quimper cancans》

so «De Kemper Cancans à Morven par Bouchaballe : Pierre Allier documentaliste et héros, le cas échéant»

\& Copies de lettres de Max Jacob à Pierre Allier.

\section{MÉDIATHÈQUE D'ORLÉANS}

Manuscrits autographes

\& «Paris-Province. Dialogues (suivi de) Petits Dialogues/Peintre et marchand » (sténographie) » [voir supra pour les publications]. $7 \mathrm{ff}$., encre noire $\mathrm{r}^{\circ}, \mathrm{n}^{\circ}$ de 1 à 7 au crayon graphite. 


\section{\& Le Terrain Bouchaballe}

Comédie en 3 actes. Tapuscrit - à l'exception du f. 2 qui donne la liste des personnages et du f. 61 -. Papier pelure avec corrections manuscrites à l'encre bleue ou noire sous pochette cartonnée marron portant le nom de l'auteur, le titre à la gouache rouge, lettres bordées de noir. Titre sur trois lignes et sous-titre. Adresse à la plume : « 17 rue Gabrielle/XVIII » barrée au profit de «St-Benoît-sur-Loire/Loiret » ajouté à l'encre. En quatrième volet de la pochette, un profil de jeune garçon à la plume. Tapuscrit composé de $67 \mathrm{ff}$. sous chemise de papier perforée (trous de classeur) portant titre et nom de l'auteur et l'indication : « La scène est à Guichen, chef-lieu de département/de nos jours. »

\& Le Journal de modes ou les ressources de Florimond

Farce en 1 acte. Note au crayon sous le titre « 10 r. Berthollet ${ }^{54} »$. Une écriture tierce au crayon charpentier a inscrit en biais " théâtre ». $54 \mathrm{ff}$. à l'encre noire au $\mathrm{r}^{\circ}$, ms au net. [voir supra pour les publications].

« Le Roi de Béotie: « Mémoires du père Vaubois », $5 \mathrm{ff}$.

Correspondances

Lettres de Max Jacob à : Armand Salacrou, Joseph Pérard [conseils pour la traduction du Piano de Chopin de Cyprian Kamil Norwid, 1937].

\section{MUSÉE DES BEAUX-ARTS D'ORLÉANS}

\section{Ceuvres graphiques de Max J $\mathbf{J}_{\text {ACOB }}$}

« Halte de comédiens dans la montagne, 1938, crayon de couleur, gouache et plume.

\& Scène de théâtre, vers 1934 ?, mine de plomb et gouache sur papier, 266 x 415.

« La Danse moderne ou l'équilibriste, 1929, encre sur papier, 271 x 165, dédicace : « La danse moderne/à Monsieur Camille Bizot/Max Jacob avril 1929. » [Cahier HT, II]

\& Parade de cirque, 1933, gouache sur papier, 349 x 500, dédicace : « À mon ami Bruneau/Max Jacob 33. »

\& Parade de cirque, 1934, gouache sur papier, 349 x 500.

« Conférence tenue à Walpurgis, allusion à Faust de Gounod, 1927, 260 x 267, plume encre noire. [Illustration page suivante] 


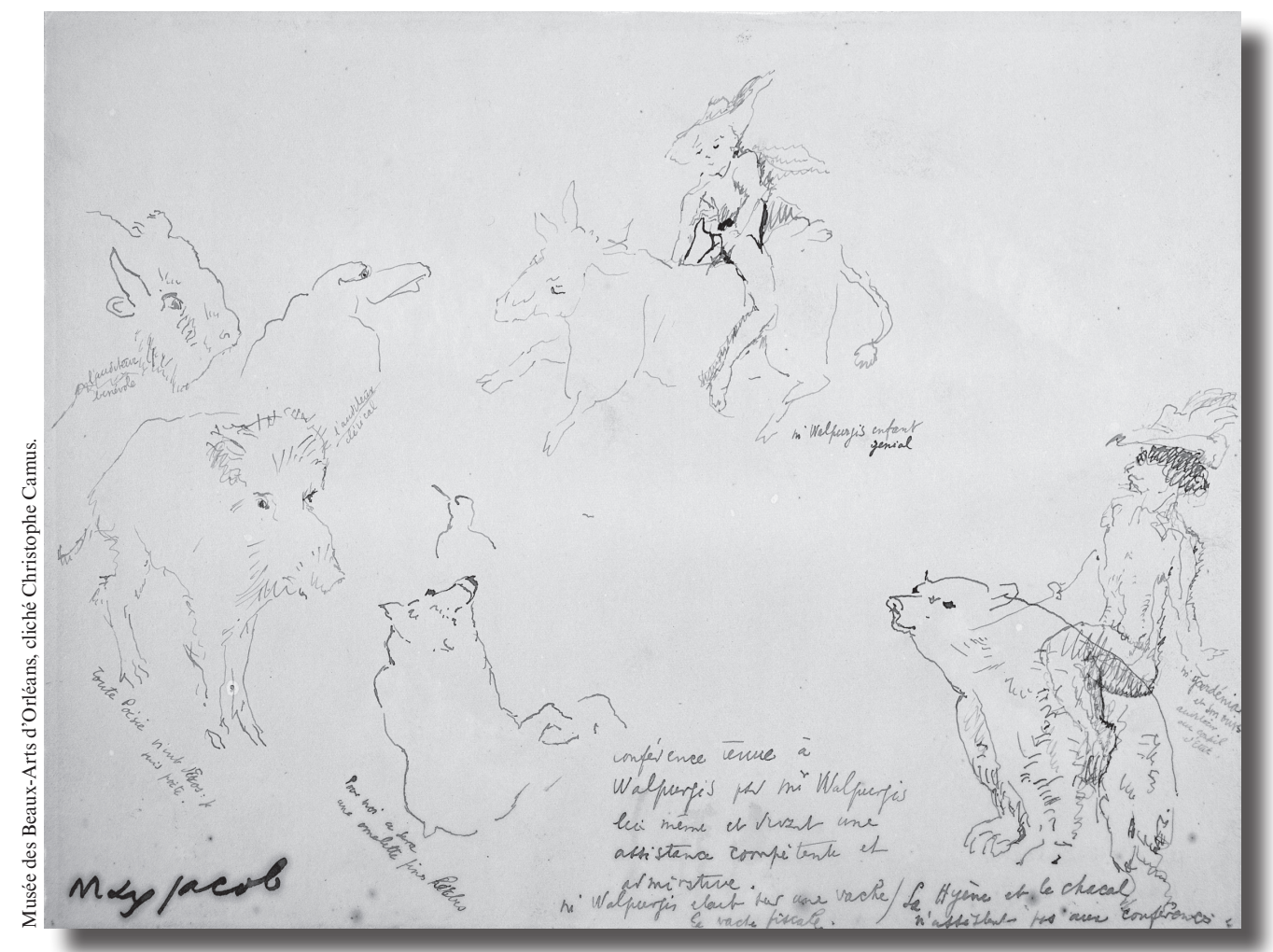

Max Jacob, Conférence tenue à Walpurgis, allusion à Faust de Gounod, 1927, 260 x 267, plume encre noire.

\& Scène de théâtre, 1922, gouache sur papier vélin, 244 x 356.

\& Le poète Orphée arrêté par les brigands, pastel.

\& La Danse de la Nymphe, 1911, papier, encre, gouache et lavis (frontispice de La Côte).

\section{CEuvres graphiques de Pierre de Belay : spectacles aux Noctambules,} 21 MARS-13 AVRIL 1936.

\&ix croquis de Marianne Oswald au fusain - Soirée Max Jacob aux Noctambules, Paris 1936

son en buste de trois quart,

sos de profil, en buste,

sos la tête rejetée en arrière, 
so la chevelure ramenée sur le front,

sos drapée dans une cape,

son en buste de face, à la robe décolletée.

\& Croquis de Jane Batori [sic], penchée en arrière, une feuille à la main, 1933 ?, crayon noir sur papier.

Photographie

\& Anonyme, Portrait de Max Jacob au piano, 1910, nb, 120 x 178.

\section{BIBLIOTHÈQUE MUNICIPALE DU HAVRE}

FDS BERnard EsDras-Gosse

\& Medrano, dessin à la plume rehaussé de gouache, 1930. [Cahier HT, XIII]

\section{INSTITUT MÉMOIRES DE L'ÉDITION CONTEMPORAINE (IMEC)}

\& Lettres de Max Jacob à : Étienne de Beaumont, Robert Caby, Erik Satie.

\section{ARCHIVES SONORES ET AUDIOVISUELLES}

Radio-Paris ${ }^{55}$ (droits perçus)

so 14 novembre 1931 (9,90 f.)

so 10 janvier 1933

sos 14 octobre 1934 (40,50 f.),

\&s 14 janvier 1937 (124f.)

so 14 avril 1939 (12,90 f.).

\& Actualités Pathé-Gaumont : www.maxjacob.com/2013/max_jacob_vid.htm Montparnasse, éditeurs, auteurs, clients fraternisent

Séance de signature du livre Chemin privé de Gaston Bonheur (éd. Jacques Haumont et $C^{\circ}$, 1933) en présence de Max Jacob, Jean Cassou et Louis de Gonzague Frick : Archives Pathé-Gaumont, $1^{\text {er }}$ déc. 1933.

\& Institut national de l'Audiovisuel, INA http://inatheque.ina.fr/ 


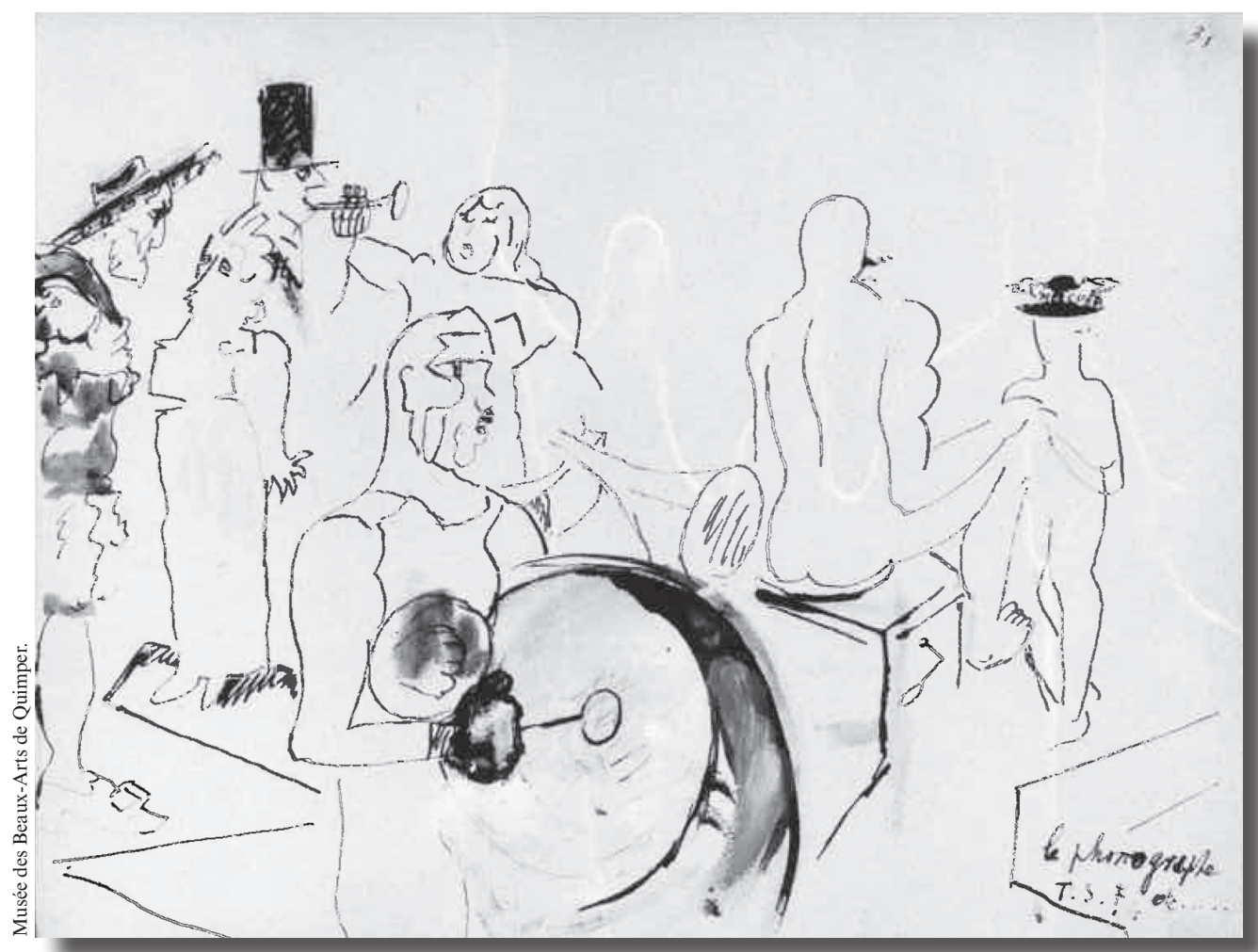

Max Jacob, « Phonographe et TSF etc... », Allusions-illusions, plume sur papier.

La base « Max Jacob» comprend :

\&) 180 notices télévisées (TV nationale) de 1951 à nos jours,

ss 389 notices télévisées (TV régionale) de 1960 à nos jours,

so 968 notices radiophoniques du 24 août 1945 à nos jours.

Notices et émissions consultables (adresses sur le site), parmi elles, les séries phares de France Musique et de France Culture se distinguent: Staccato, Poésie sur Parole, Comédie-Française, Max Jacob et ses musiciens.

\section{SÉRIES TÉLÉVISÉES}

$\propto$ Les Aventuriers de l'Art Moderne $e^{56}$

Documentaire en six épisodes d'après un scénario de Dan Franck, réal. Amélie Harrault, Pauline Gaillard et Valérie Loiseleux, Arte France/Silex Films. Musique Pierre Adenot ; voix Amira Casar. Première diffusion Arte (16, 17, 18 déc. 2015), disponible en DVD. Le premier épisode est consacré à 
Max Jacob, les épisodes suivants évoquent l'auteur à plusieurs reprises.

\& Genius, saison 2 dédiée à Pablo Picasso, National Geographic/FOX 21 Television/Studios \& Imagine Television. Première diffusion USA, 25 avril 2017. Distribution : Clémence Poésy : Françoise Gilot; Alex Rich : Picasso jeune ; Antonio Banderas : Picasso ; T. R. Knight : Max Jacob.

\section{LONGS MÉTRAGES}

« COLOMO Fernando (réal.), La Banda Picasso : Arti Film, 2012, 2 h. Musique : Juan Bardem, scénario Fernando Colomo. Distribution : Lionel Abelanski : Max Jacob, Ignacio Matéos : Picasso, Raphälle Agogué : Fernande, Pierre Bénézit : Apollinaire.

\& AGHION Gabriel (réal.), Monsieur Max ${ }^{57}$, scénario de Dan Franck, Films en Stock/ARTE France/S.F.P./France 3-C.N.C-ANGOA-PROCIREP et le soutien de la région Île-de-France, 2007, disponible en DVD. Distribution : Jean-Claude Brialy : Max Jacob, Guillaume Gallienne : Max Jacob jeune, Féodor Atkine : Picasso, Jean-Claude Dreyfus : Guitry, Dominique Blanc : Alice.

« DAVIS Mick (réal.), Modigliani, dir. photographie : Emmanuel Kadosh ; musique : Guy Farley, Limelight distribution/Lucky 7/LLC, 114 mns, 2004, disponible en DVD. Distribution : Udo Kier : Max Jacob, Andy Garcia : Modigliani, Elsa Zylberstein : Jeanne Hébuterne, Hippolyte Girardot : Maurice Utrillo, Omid Djalili : Picasso.

\section{DOCUMENTAIRES}

\& KELNER Vincent (réal.), Max Jacob, Drancy 2004, scénario de José Cruz, production Colle/Cruz/AMJ, 1 h., 2004.

\& ANDREU Anne, FERRARI André, Max Jacob, coll. Un siècle d'écrivains, $n^{\circ} 9$ : France 3/Ciné TV/INA/, 47 mns, 1995. Commentaire : Anne Andreu ; réal. : Alain Ferrari ; voix : Christophe Allwright, Michel Bouquet.

« TRÉHOREL Denis, Max Jacob: Agence Culturelle de Bretagne. Colloque de Vannes Qui (ne) connaît (pas) Max Jacob/Université du $3^{\mathrm{e}}$ âge et Pour Tous, 50 mns, 1987. 


\section{CONSULTABLES SUR LE SITE DE L'ASSOCIATION DES AMIS DE MAX JACOB}

\& SUSTRAC Patricia, Max Jacob 1940-1944 : Une Histoire de vie. Scénario et voix : Patricia Sustrac, 5 mns, Agence Anamnésia : Ville d'Orléans, 2014.

\& SUSTRAC Patricia, Fonds Max Jacob en Région Centre, scénario et voix : Patricia Sustrac, Agence Richard Delaume, production CICLIC/Région Centre/Ministère de la Culture, 3,32 mns, 2013.

\& CLUZEL Raphaël, Max Jacob un jeune poète de 100 ans (diff. 11 nov. 1977 à $19 \mathrm{~h} \mathrm{05}$, France 3), 6 mns.

« ANONYME, «L'Enterrement de Max Jacob à Saint-Benoît-sur-Loire, 5 mars $1949 », 1949$.

\section{CONSULTABLE EN SALLE D'EXPOSITION DU MUSÉE DES BEAUX- ARTS, QUIMPER}

« ALLARD Pierre, CHEVRIÈRE Emmanuelle, Max Jacob, Coup d'œil/INA/ Direction du Livre et de la Lecture (Ministère de la Culture), 1994, 26 mns.

\section{NOTES}

1 Jacob est admis à la Société des auteurs (aujourd'hui SACD) en septembre 1921 (dossier 517) et à la Sacem en août 1933, Darius Milhaud et Jacques Leyrolles sont ses parrains. Jacob est représenté à la Société des auteurs par l'agence d'Alfred Bloch puis Besnard à partir de 1940, Bloch étant licencié en raison de ses origines juives. La fiche CARDEX récapitule l'ensemble des droits d'auteurs versés à Jacob depuis son affiliation. Au 14 mars 1941, le solde créditeur est de 115,20 f. Le 25 mai, la somme est virée au compte de Maxime Jacob, musicien, homonyme du poète. Il n'y a pas de droits enregistrés à la Sacem à partir de 1940, puisque Jacob visé par les mesures antisémites est interdit de publications et de représentations.

2 André Malraux a reçu 150 frs au titre de directeur de la coll. (Binoche et Giquello, 8 février 2017, lot 127).

3 Nombreuses variations avec le texte paru dans Nord-Sud.

4 Dans une lettre à Paul Fort, Jacob évoque un projet théâtral commun : « Paris, Ermitage du Régent, 7 juin 1928/ Ton nom est bien doux à mon souvenir et nous avons eu de belles nuits dont tu étais l'ornement. Te souviens-tu que nous aimions trois vieilles opérettes, que nos mères nous avaient apprises. Une fois le matin nous surprit en train d'écrire un drame "plus beau que Shakespeare" », (Pierre Bergé, 6 juin 2012, lot 48).

5 Le 9 décembre 1937, Jacob signale être l'auteur d'un poème que Sylvette Fillacier veut 
transposer $(P J, 441)$. En note Didier Gompel indique que la comédienne avait sollicité Jean Marèze, frère cadet de Carco. Est-ce une autre transposition ?

6 Dossier Sacem. Éditions et traductions approuvées par Jacob le 14 février 1939.

7 «Tu pâlis, tu palpites, tu trembles, tu laisses tomber tes fleurs. Ne sommes-nous pas ensemble? Ton front n'est-il pas sur mon cœur? Devant l'ostensoir de tes tresses des branches se courbe la proue. Quand le jardin dit une messe au triste autel de nos genoux sous le doux vent de l'automne les feuilles s'écartent pas à pas, se guettent, se questionnent, s'offensent de ton embarras. Dîtes-moi si je suis folle ? Entre le porche et le taillis, j'ai vu passer le fantôme de celui que j'ai trahi. Mon époux est à la guerre, taché de sang qu'elle a bu. Quand je vous aime, on l'enterre. Adieu, je ne vous verrai plus, Adrien (3 fois). »

8 « [J'attends la lune au coin des places. J'attends la lune au coin des mots. J'attends la lune au fond des glaces, j'attends la fin de mon repos.] Je suis un cadavre d'infirme sur le plancher d'un atelier. Et mes yeux sont des tirelires ou des livres morts sont liés. Emportez la mort et la vie, emportez le bonheur, emportez la gloire et l'envie, les espoirs et les désespoirs. Emportez la nappe et les chaînes, emportez la chute du jour. Je suis esclave et je suis reine. On dira qu'il est mort d'amour ». Reprise de [à].

9 «Vos yeux clos, votre main lasse, votre main pâle qui passe sur le cadran solaire d'ardoise. Les plis d'un lourd manteau de soie. L'anneau mystérieux à vos doigts, les pigeons bleus sur le toit. Les fronts des arbres qui se posent l'un sur l'autre, l'odeur des roses et le soir qui se repose. Le poème des astres morts. La tristesse pour un peu d'or et mon amour pour vous Léonor !»

10 " "Vous n'irez plus au bal, vous êtes la dernière". Les conscrits le chantaient sur la charrette à bras. [Vous n'irez plus au bal, Madame la fermière. Vous n'irez plus au bal si ce n'est le lundi. Vous n'irez plus au bal, vous irez dans mon lit.] Caravelle de mes rêves, nous n'avons plus un carat mais écoutez la nouvelle, on te joue à l'Opéra ! Un astre est en courroux. Il se paye nos têtes, s'il croit nous rendre fou, il faut qu'il soit bien bête. » Reprise de [à].

11 Voir infra l'article de Florence Collin et la correspondance croisée inédite de Max Jacob avec le vicomte et la vicomtesse de Noailles (1929-1934).

12 Idem. Cantate reprise les 2 et 3 avril 2012 au Théâtre de Hyères devenu le Théâtre Denis, orchestre dirigé par Patrice Lartigue, solistes : Catherine Decaen et Luc Coadou. Manifestation organisée par La Villa Noailles.

13 « Je te remercie de la musique si grande et si émue dont tu as embelli mes pauvres vers. Je t'en félicite [...] Remercie aussi la très bonne cantatrice qui a bien voulu s'intéresser à nous. Elle est admirable et belle. C'est dans tous les sens du mot : une belle artiste. Bravo à tous les deux, quelle voix ! », lettre inédite à Virgil Thomson, 16 juin 1931. La lettre est constellée en tous sens de «Bravo ! ", (Université de Yale, Virgil Thomson papers, ms 29 à ms 29 A Box 54, folder 20 [Jacob Max 5-0-4]).

14 Voir infra la correspondance revue et augmentée de Max Jacob à Roland-Manuel.

15 Reprise dans une mise-en-scène de Paul-Émile Deiber, chorégraphie de Jacques Chazot et scénographie de Jean-Pierre Ponnelle à l'Opéra-Comique le 22 mai 1959.

16 Le projet de cette " petite parodie [...] avec musique " a été retardé faute de trouver un producteur (à Doucet, GI, 176) et finalement, abandonné. Milhaud a donné un compte rendu dans Le Courrier musical regrettant qu'on " ne porte pas en scène ce poème symphonique d'une construction et d'une écriture d'orchestre toutes classiques ", (MILHAUD Darius, Notes sur la musique, essais et chroniques, textes présentés par Jeremy Drake, Flammarion, coll. Harmoniques, 1982, p. 56) :

Au premier tableau, le mystère de Max Jacob nous fait assister d'abord au dialogue mystique entre la Sainte et le Bien-Aimé au nom duquel répond l'Ange. Elle a fait vœu de silence que 
les sœurs du couvent protègent. Avant de disparaître, l'Ange la met en garde contre un jeune homme dont elle attend la visite. Après un bref dialogue de la Sainte et d'une sœur s'inquiétant de ses yeux trop brillants de fièvre, la scène demeure vide. Entre le jeune homme dévoilant ses noirs desseins : perdre à tout prix la Sainte. Il se retire, la Sainte réapparaît troublée par des parfums où elle ne reconnaît pas la présence de son Bien-Aimé.

Rideau et interlude orchestral séparant les deux tableaux.

Dans la cellule, confrontation entre le jeune homme et la Sainte : il se fait passer pour un saint confesseur et tente de l'arracher à la clôture en l'incitant à venir purger le pays de la peste qui l'accable puis, lorsqu'elle résiste, en lui révélant que son frère en est déjà mort et que sa mère agonise : elle seule peut la sauver. Soudain on voit apparaître la Vierge qui transforme le jeune homme en animal. L'Ange annonce à la jeune fille que ses épreuves sont terminées et que la Vierge elle-même vient la conduire vers Dieu. L'Apparition s'efface, la sœur pénétrant dans la cellule assombrie y trouve Alméenne évanouie et invite ses compagnes à se joindre à elle : «Sainte Alméenne, priez pour nous » cependant que le rideau tombe.

17 Des décors ont sans doute été envisagés par Guy-Pierre Fauconnet. Nous remercions Caroline Manceau, auteur d'un mémoire sur l'artiste pour le Musée des Arts décoratifs de Paris de cette information. L'opéra a été repris et complété dans l'esprit d'Honegger par Nicolas Bacri à la demande du festival Vredenburg et Transparant, Utrecht le 26 novembre 2005.

18 Le 19 avril 1923, Max Jacob confiera à Jane Bathori « qu'il n'a guère entendu la musique de Ste Alméenne et qu'[il] en est navré » (BnF, dpt Musique, BOB 28 660, f. 177-178);

19 Revue pamphlétaire fondée en 1933 par Marcel Pagnol. Jacob a été sollicité par Gabriel d'Aubarède.

20 Revue éphémère du couturier et costumier parisien Jacques Heim (1899-1967).

21 Jacob a inscrit sur ses souches comptables : «Films (article) : $100 »,($ Souches comptables ayant servi de palette, MBAQ, inv. D-50-1-80).

22 Jacob précise à Bernard Esdras-Gosse les textes qu'il déclame : « des lettres du Cabinet noir, des poèmes du Cornet à dés et quelques poèmes très lyriques et très graves ", (Lettres à Bernard Esdras-Gosse, éd. Seghers, 1953, 29 mars 1936, p. 36).

23 Voir le récit désabusé de la soirée à René Dulsou ( $A A I$, p. 302-303).

24 Jacob adressera à Jane Bathori un pneumatique qui exprime toute son admiration : « À ma sublime Bathori, merci/à ma vaillante, à ma vivante amie./À ma haute musicienne/à ma voix si souple/à ma comédienne adorable/à mon intelligente et géniale artiste/à ma belle, à ma bonne et/fine Bathori/Merci ! Max Jacob » (4 avril 1933, BnF, dpt. de la Musique, BOB 28 660).

25 «Cher ami/Je ne vous ai pas encore dit combien mon pauvre cœur est touché de ce que vous faites pour lui. Ainsi mes dialogues ont été lus, joués sur une scène - et grâce à vous. Comme c'est encourageant et comme parfois une raie lumineuse apparaît entre les battants de la porte close de l'Olympe ! », lettre inédite de Max Jacob à Julien Lanoë, s. d, 55 rue Nollet (BM Nantes). La représentation est enregistrée à la Société des Auteurs le 26 mai 1930. Six représentations ouvrent droits à répartition. Le texte a été confié à Cliquet-Pleyel qui composera une mélodie, mais ne trouvera pas d'acteurs (lettre inédite, 26 février, coll. Gaspard Olgiati, « Lettres reçues par Max Jacob, 1929-1930»). Le ms est perdu. Sur le bordereau d'admission à la Société, Jacob déclare Le Kleptomane comme opérette «non éditée, jouée en partie au Théâtre de l'Atelier » (bordereau 13688, 22 août 1933). Une lettre inédite de Cliquet-Pleyel à Max Jacob sollicite de l'auteur un résumé circonstancié pour introduire la forme du sketch, le texte de Jacob a été diffusé par hautparleur comme s'il s'agissait d'une conversation téléphonique (coll. Gaspard Olgiati, op. cit.)

26 Les Matinées sont créées en 1920. La Bibliothèque de la Comédie conserve un dossier de huit ff. de poèmes de Max Jacob. 
27 La programmation s'interrompt en 1940 : la Comédie-Française ne distribue pas les auteurs juifs (voir JOUBERT Marie-Agnès, La Comédie-Française sous l'Occupation, Tallandier, 1998, p. 146-148).

28 Carton d'invitation dans $A A I$, p. 114. Jacob a encaissé la somme de $21^{\mathrm{f}}$ en janvier 1923 (Souches comptables, op. cit.).

29 Le 7 février 1922, Le Gaulois informe que : « Charles Dullin dont on connait les efforts et les tentatives va présenter trois spectacles donnés par sa nouvelle école du comédien : l'Atelier. On jouera L'Avare avec un dialogue improvisé [...]. Enfin Chantage de M. Max Jacob » (n 16197, p. 4). Voir également $M J J C$, p. 78 et $80 \mathrm{n} .1$ et 2, Jacob note la programmation en matinée.

30 Pierre Bertin est alors pensionnaire de l'Odéon. Le 24 mai Comœdia informe que « La curiosité de ce spectacle [quatre comédies-bouffes d'un esprit très nouveau] vient de ce qu'il est en grande partie dû à des poètes [...]» (anonyme). Cocteau donnera un bref compte-rendu du spectacle : « [...] organisé généreusement par Pierre Bertin au Théâtre Michel (je dis généreusement, car à l'encontre de ce qu'imaginent certains chroniqueurs naifs, il doit y être de sa poche) lui vaut toute notre gratitude. Il a, en effet, choisi et montré des ouvrages dont la valeur ne peut apparaître qu'à un petit nombre de personnes. Sa récompense est d'avoir réuni tous les suffrages comme interprète. Ce n'est pas à moi de parler de La Femme fatale, où la poésie parodique et la poésie véritable s'enchevêtrent [...]», (Comøedia, 28 mai 1921, rubrique « Excuses aux critiques »).

31 «La piécette de Max Jacob dont chaque scène nous paraît d'un comique irrésistible, écrira Georges Auric -, [...]. Elle nous avait semblé, dans sa lecture, irrésistible. Irrésistible aussi les répétitions [...] Un fragment de sa conclusion me reste toujours présent, avec ses airs de mirliton : "Madame, à l'aventure/Errant sous les tilleuls/A perdu sa coiffure./Monsieur rentre tout seul./Ah ! cueillir la noisette/Est un plaisir bien doux/aveque [sic] sa Lisette/Quand chante le coucou... (...)/coucou, coucou, coucou... Caillou, choux, genoux, hibou - et je le crains :/ Cailloux d'choux" ", (Le Potomak, 27 mars-1 ${ }^{\text {er }}$ avril 1990, p. 8-9).

32 On note $106^{\mathrm{f}}$ sur les souches comptables de Jacob en juin 1922 (op. cit.). L'auteur aurait-il cédé le ms ? Aucune indication sur l'acheteur n'est notée. Les variations du titre peuvent-elles être liées à l'ouvrage homonyme d'André Birabeau publié chez Stock en 1922 ? Est-ce un mot «à la mode » ? Voir Mireille Dottin-Orsini, Ces femmes qu'ils disent fatales. Textes et images de la misogynie fin-de-siècle, Grasset, 1993. Le titre éloquent nous renseigne sur cette passion dont le siècle se délecte au théâtre comme au cinéma.

33 Le Piège de la Méduse de M. Erik Satie, avec musique de danse du même Monsieur, orné de trois gravures sur bois par Georges Braque : Galerie Simon, 25 juin 1921.- rééd. avec gravures de Georges Braque, Pantin : Le Castor astral, coll. Les Inattendus, 1988. Pierre Bertin interprétait le rôle-titre à la création.

34 RADIGUET Raymond, Les Pélican, avec sept eaux-fortes d'Henri Laurens : Galerie Simon, 1921. - rééd. sans ill. dans RADIGUET Raymond, Euvres complètes, Omnibus, 2012, p. 228-243.

35 Ibid., p. 262-276.

36 Jacob confirme la perte du manuscrit à Robert Guiette en 1928 (Lettres à Robert Guiette : éd. des Cendres,1996, p. 70). Le compte rendu de Cocteau, "Max Jacob dramaturge » est la quatorzième chronique de la série Carte blanche (Paris-Midi, 30 juin 1919, $9^{\mathrm{e}}$ année, $\mathrm{n}^{\circ}$ 2317, p. 3). - rééd. Carte Blanche, La Sirène, 1923 (reprise avec ill. de l'auteur chez Mermod, Lausanne, 1953) :

Max Jacob a connu un gros succès avec sa pièce bouffe en un acte. [...] Succès double de dramaturge et d'interprète, car Max Jacob tenait lui-même le rôle du proviseur. La scène se déroule dans un collège de province. Un scandale éclate : l'élève et la fille du proviseur. Un autre: l'épouse du proviseur et le censeur. Le proviseur rapporte ces scandales. Il a une bonne 
formule: "Les femmes au gynécée, les hommes au forum. "Il entraîne le censeur prendre l'apéritif. Mais l'élève est un " anarchiste», la mère et la fille veulent vivre en beauté. Ces trois grandes âmes quittent le collège pour figurer au théâtre de Nantes. On raconte mal Guignol. Guignol est excellent par hasard. Guignol exprès est plus difficile. Le réussir est un tour de force. Max Jacob, sa canne, sa calotte, sa tabatière, ses menaces, ses larmes de peur, fut un guignol en bois et en lustrine. Mais la poupée de guignol est un gant, et il y avait sous le gant une main d'une finesse merveilleuse.

37 Je remercie le collectionneur Alexandre Eltchaninoff de m'avoir communiqué ce programme très rare.

38 Une carte postale inédite d'Henri Sauguet à Max Jacob évoque « la joie de penser que nous verrons bientôt notre cher Titien en chair et en os !» (10 août 1934, coll. Marie-Lionèle Floch). Le 5 août, Marcel Herrand remercie Jacob de solliciter Picasso pour les décors (lettre inédite, idem).

39 Lettre à Jean Paulhan, [mai ou juin 1930], MJJP, p. 158 et lettre à Madame Aurel (PJ, p. 331) : « [Dullin] aurait tiré un misérable sketch d'une grosse opérette en 4 actes ». Cependant, nous ne trouvons pas de programmation sur les bordereaux de saison (fds Charles Dullin, Dpt. des Arts du spectacle, BnF).

40 «J'envoie Bouchaballe pour le voir exprimé » (Lettres aux Salacrou, août 1923-janvier 1926 avec un portrait d'André Beaudin, Gallimard, 1957, p. 30-32, 16 janv. 1924). Le ms a été retrouvé et publié posthumément (voir infra). Joseph Quesnel (1897-1931) est le fondateur du mouvement culturel coutançais Le Pou qui grimpe et de la revue Le Chien de pique (1927-1931) à laquelle Jacob livrera des dessins.

41 Le Grenier de Toulouse et le Théâtre national de Chaillot ont diffusé simultanément un spectacle de Didier Carette intitulé Le Cabinet noir, montage de textes de Max Jacob.

42 Voir infra p. 491-492.

43 Pour les thèses musicales, consulter www.theses.fr ou www.sudoc.abes.fr

44 BnF 28312 (760). Le directeur de thèse n'est pas spécifié. Je remercie Alain Andreucci pour son aide.

45 Une exposition réalisée par les élèves de la classe des métiers de la culture musicale et un concert accompagnaient la journée d'étude.

46 Compte rendu de l'exposition de la BnF, 14 juin-22 juillet 1995 dont « le clou de l'exposition » était constitué par «le ms des Quatre poèmes de Max Jacob dédié à Darius Milhaud accompagné d'une lettre de Jacob à Milhaud du 31 décembre 1931: «Ce serait mon seul titre de gloire de figurer à côté de ton nom. »

47 La pièce est restée inachevée, elle croise deux thèmes fréquents chez Jacob : la paternité inassouvie et le sort des enfants naturels (voir L'Homme de chair et l'homme reflet, Un amour $d u$ Titien). L'action se déroule sur fond de guerre avec les îles de la mer Egée. La pièce est truffée de chœurs, de musiques militaires et de déclarations patriotiques pastiches d'Hervé. Alexis et Victor sont deux frères amoureux de Lolotte (alias Charlotte ou Ginette Corday), employée d'une grande banque internationale qui veut partir faire du cinéma à Yokohama (act. I). Alexis veut reprendre l'enfant qu'ils ont eu ensemble, Angela, mais est-il vraiment le père ? Ne serait-ce pas plutôt Victor? Si Victor est le père, Alexis est-t-il l'oncle du fils de sa femme ? «Serait-ce des amours pédérastiques » (act. I, sc. 1) ? Alexis, mobilisé confie l'enfant à une certaine Mme Plon qui l'envoie à «l'Asile des Petits Éplucheurs orphelins. » À son retour, Alexis épouse Lolotte, retrouve l'enfant mais est-ce le vrai car il a régné un grand désordre à l'Asile et il n'y a pas de papiers pour prouver l'identité de ceux qui se présentent devant Alexis devenu un grand producteur de cinéma. Arrive un jeune garçon qui se révèle être leur enfant et, à sa vue, Angela, fille de Lolotte d'un précédent mariage, tombe amoureuse de lui. Or Angela 
est sa demi-sœur : le mariage est impossible. On lira une situation identique dans « Le crime de Honfleur » dans Le Phanérogame.

48 La pièce est marquée par un fort contenu autobiographique. Une mère et ses trois enfants : Philippe, Dejanire et Antoine, "pataud, et lourd d'esprit ». Antoine est lent, un peu nigaud, il inquiète sa mère car « il a des polypes dans le nez ». Une fée entre dans la chambre d'Antoine : en rangeant des papiers, elle se coupe un doigt (sc. 2). Les enfants rentrent de l'école : Antoine est la risée de tous car il ne sait pas délacer ses souliers, il pleure en silence (sc. 3). La fée délace les souliers d'Antoine, examine ses leçons et l'aide pour sa version et son thème latin. Elle l'interroge : Histoire de France et L'Hymne à l'esprit de Brizieux. Elle souffle sur les yeux de l'enfant, le touche de sa baguette magique. Antoine récite sans hésitation. Qui est-elle ? elle est une fée qui a eu pitié de lui " car il est un bon petit garçon », elle s'appelle Ariane. Antoine est ravi : "Quel bonheur, je vais être toujours le premier, maman ne me grondera plus et personne ne se moquera plus de moi » (sc. 4). Antoine stupéfie ses frères et ses sœurs, il sait toutes ses leçons. Personne ne croit qu'une fée est venue. La mère pense qu'Antoine n'a pas fait son devoir tout seul et que Virginie, la femme de chambre, en est l'auteur. Elle le trouve fatigué et va lui faire une infusion des familles : Antoine «Tout le monde me fait des misères ici. Je suis un enfant martyr. Mais elle (la fée) me vengera » (sc. 5 et 6). On appelle le docteur ; la mère a peur car «Antoine a des visions »; le médecin relativise : "Les cas de mensonge sont fréquents à l'âge de la croissance, un de mes confrères a fait une thèse sur le sujet : "Les petits menteurs devant la faculté" ». Le docteur cite Charcot. Il examine l'enfant et diagnostique des troubles mnésiques : «Tu lis beaucoup de romans : Jules Verne...» (sc. 7). Cependant la fée est sur le balcon, les enfants la voient (sc. 8 à 11). Sc. 12 : la mère cite sa belle-sœur : Mme Iklé. Le docteur prescrit un remède pour toute la famille : hydrothérapie et gymnastique suédoise. La fée descend lentement au bord de la scène et le rideau tombe. On reconnaîtra l'épisode biographique du séjour du petit Jacob dans un institut de santé, le personnage de Mme Iklé (Chantage) et le remède d'une époque friande d'hydrothérapie (voir Isabelle et Pantalon).

49 Ms publié dans DICKOW Alexander, Jacob et le cinéma, op. cit., p. 101-104. Le ms autographe que nous avions relevé pour cette publication était, à l'origine, un des dix-sept chapitres de $L a$ Fille du garde-barrière, roman à paraître en 1923 aux éditions Paul Budry et Henri Mermod. Jacob avait dressé le plan et dessiné la maquette mais ce projet ne vit jamais le jour. L'avantprojet du roman (chapitres, nombre de signes par chapitre...) est conservé à la BnF (NAF 28312-6). Dans une lettre de René Auberjonois à Charles A. Cingria circa avril-juin 1927, Max Jacob demande qu'on transmette ses : « [...] amitiés à Budry [qu'il] ne cesse pas d'aimer -. [Il] le supplie de ne jamais faire paraître le piètre manuscrit qu'il a de [lui]. » Auberjonois demande à ce que ces propos ne soit pas transmis «à Mermod qui est capable de prendre cette modestie à la lettre. La Petite Garde Barrière est une vraie sublimité. » (CINGRIA C.- Albert, Cuvres Complètes, Lausanne : L'Âge d'Homme, 1981, vol. XVII, p. 308-310). Je remercie J.Christophe Curtet (association des Amis de Cingria) de cette référence.

50 Ces lettres proviennent des lots de lettres confiées par Max Jacob à Michel Manoll en 1938 pour être vendues, Jacob s'est ensuite rétracté suites aux réactions de ses destinataires alertés par la presse. Le catalogue de cette vente a cependant été publié, voir Philobiblion, bulletin périodique de beaux livres, livres et autographes, Librairie Lipschutz, XLVIII ${ }^{\mathrm{e}}$ année, $\mathrm{n}^{\circ} 72$, 1938, lots 491-493.

51 Les personnages sont hauts en couleurs : Fricassard, Monsieur Pluguffan - « ancien receveur de l'impôt sur les casquettes », Madame Bannalec, des commères et des bonnes, la ville de Quimper, la Tour d'Auvergne.... Il y a pléthore de personnages. Il y est question du temps, de la politique, de la nationalisation des terres et de la suppression de l'impôt (Max Jacob était-il un adepte de 
Léon Walras ?), de la Révolution, des abris d'hygiène - Ubu n'est pas loin et chatouille le goût pour la scatologie des auteurs. On y entend L'Internationale, les revendications de Réo - ouvrier socialiste - et sur l'air du Bon roi Dagobert la complainte des facteurs : « Les facteurs de Quimper/ ont toujours le feu au derrière./Ils font leur service/C'en est épatant/Comm' les écrevisses... » Le final appelle au patriotisme des bretons pour reconquérir l'Alsace et la Lorraine (on est en 1910) ! Le producteur à la lecture de ce texte surprenant, prudent, avait sollicité l'avis des édiles, le retour acheva de persuader de la folie du projet.

52 Max Jacob a fait don à la Bibliothèque de Quimper d'un carton contenant les coupures de presses le concernant relevées par l'Argus auquel il était abonné.

53 Dossier Sacem ouvrant droit à la répartition de 36 parts (23 janvier 1935).

54 Lors des représentations d'Isabelle et Pantalon, Jacob a logé chez Dubuffet, 10 rue Berthollet (15 déc. 1922- 4 janv. 1923). Il est probable que la farce ait été inspirée et remaniée à partir d'une autre pièce intitulée La Leçon de diction, pièce en un acte. Jacob a adressé ce scénario « très développé qui met en scène Florimond Galuchard, professeur de diction, aux prises avec une alsacienne et un muet » à un destinataire non identifié le 21 septembre 1922. Jacob joint à La Leçon deux autres scénarii non titrés : le second est un «"bouffe" qui verra la séduction de trois personnages : un poète, une actrice et sa bonne », le troisième est une " histoire orientale » (Bibliothèque d'un amateur, surréalisme, dadaïsme, cubisme, livres et manuscrits, dessins et illustrations, reliures, 23-24 mars 1981, lot n²12).

55 Les archives de Radio-Paris sont fragmentaires et pour la plupart détruites soit volontairement - cette radio ayant été, durant la guerre, un outil de propagande - soit accidentellement. Les Cahiers de Radio-Paris ne conservent pas non plus de traces des conférences de l'auteur.

56 Compte rendu de Jean-Marc Pontier, CMJ 17/18, p. 379-381.

57 Voir Lettres et Mots, supra. 